\title{
MicroRNA-21 Mediates Angiotensin II-Induced Liver Fibrosis by Activating NLRP3 Inflammasome/IL-1 $\beta$ Axis via Targeting Smad7 and Spry1
}

\author{
Zuo-Wei Ning, Xiao-Ying Luo,, Guo-Zhen Wang,, Yang Li,, Miao-Xia Pan, Ren-Qiang Yang, \\ Xu-Guang Ling, Shan Huang, Xiao-Xin Ma, Si-Yi Jin,, Dan Wang, and Xu Li, ${ }^{1}{ }^{1}, 4$
}

\begin{abstract}
Aims: Angiotensin II (AngII), a vasoconstrictive peptide of the renin-angiotensin system (RAS), promotes hepatic fibrogenesis and induces microRNA-21(mir-21) expression. Angiotensin-(1-7) [Ang-(1-7)] is a peptide of the RAS, which attenuates liver fibrosis. Recently, it was reported that the NOD-like receptor family, pyrin domain containing 3 (NLRP3) inflammasome participated in liver fibrosis. However, it remains unclear how mir-21 mediates AngII-induced NLRP3 inflammasome activation. We investigate the role of AngII-induced mir-21 in the regulation of NLRP3 inflammasome/IL-1 $\beta$ axis in liver fibrosis.

Results: In vivo, circulating mir-21 was upregulated in patients with liver fibrosis and was positively correlated with liver fibrosis and oxidation. Treatment with Ang-(1-7) inhibited mir-21, NLRP3 inflammasome, and liver fibrosis after bile duct ligation (BDL) or AngII infusion. Inhibition of mir-21 suppressed the Smad7/Smad2/3/NOX4, Spry1/ERK/ NF- $\kappa$ B pathway, NLRP3 inflammasome, and liver fibrosis induced by AngII infusion. In vitro, AngII upregulated mir-21 expression via targeting Smad7 and Spryl in primary hepatic stellate cells (HSCs). In contrast, Ang-(1-7) suppressed mir-21 expression and oxidation induced by AngII. Overexpression of mir-21 promoted oxidation, and collagen production enhanced the effect of AngII on NLRP3 inflammasome activation via the Spry1/ERK/NF- $\kappa \mathrm{B}$, Smad7/Smad2/3/NOX4 pathways. However, downregulation of mir-21 exerted the opposite effects.

Innovation and Conclusions: Mir-21 mediates AngII-activated NLRP3 inflammasome and resultant HSC activation via targeting Spryl and Smad7. Ang-(1-7) protected against BDL or AngII infusion-induced hepatic fibrosis and inhibited mir-21 expression. Antioxid. Redox Signal. 27, 1-20.
\end{abstract}

Keywords: microRNA 21, angiotensin II, angiotensin-(1-7), NLRP3 inflammasome, reactive oxygen species, liver fibrosis

\section{Introduction}

$\mathbf{L}$ IVER FIBROSIS, WITH excessive extracellular matrix deposition, is a common stage of chronic liver diseases. Hepatic stellate cells (HSCs) play a key role in liver fibrogenesis in response to profibrogenic mediators (10).

MicroRNA-21 (mir-21) has been shown to promote fibrogenesis in cardiac muscle (38), kidneys (46), and lungs

\section{Innovation}

AngII-induced mir-21 activates NLRP3 inflammasome in hepatic stellate cells (HSCs) via targeting Spryl and Smad7, resulting in HSC activation. Ang-(1-7) protected against bile duct ligation or AngII-induced liver fibrosis and inhibited the expression of mir- 21 .

\footnotetext{
${ }^{1}$ Guangdong Provincial Key Laboratory of Gastroenterology, Department of Gastroenterology, Nanfang Hospital, Southern Medical University, Guangzhou, China.

Departments of ${ }^{2}$ Respiratory Diseases and ${ }^{3}$ Emergency Medicine, Nanfang Hospital, Southern Medical University, Guangzhou, China.

${ }^{4}$ State Key Laboratory of Organ Failure Research, Guangdong Provincial Key Laboratory of Viral Hepatitis Research, Department of Infectious Diseases, Nanfang Hospital, Southern Medical University, Guangzhou, China.

(c) Zuo-Wei Ning, et al., 2017; Published by Mary Ann Liebert, Inc. This article is available under the Creative Commons License CC-BYNC (http://creativecommons.org/licenses/by-nc/4.0). This license permits non-commercial use, distribution and reproduction in any medium, provided the original work is properly cited. Permission only needs to be obtained for commercial use and can be done via RightsLink.
} 
(20). Moreover, recent studies indicated that mir-21 induces liver fibrosis by activating HSCs $(12,48)$. Smad7, an inhibitory Smad that downregulates the TGF- $\beta /$ Smad pathway, is a target of mir-21 (20). In addition, the ERK-negative regulator Spry1, also a target of mir-21, mediates the effects of mir-21 through augmenting ERK kinase activity in cardiac fibroblasts (38) and HSCs (49). Hence, mir-21 stimulates the Smad, ERK pathway by promoting Smad7 and Spryl degradation. However, the potential link between these two target genes during mir-21-induced HSC activation remains unclear and needs further investigation.

Recently, the NOD-like receptor family, pyrin domain containing 3 (NLRP3) inflammasome complex (including NOD-like receptor NLRP3, the adaptor molecule apoptosisassociated speck-like protein containing a caspase-recruitment domain (ASC), and the effector molecule procaspase-1) has been revealed as the novel mediator of liver fibrosis (36). It is believed that NLRP3 activation causes assembly of the NLRP3 inflammasome, leading to activation of caspase-1, which cleaves pro-IL- $1 \beta$ and pro-IL-18 to their maturational forms (34). The NLRP3 inflammasome has been reported to amplify chronic liver inflammation and activate HSCs (39, 41). The NLRP3 inflammasome is a promising target for treatment of hepatic fibrosis.

NLRP3 inflammasome activation includes a two-step process: the first signaling step termed as the signal 1 : NF- $\kappa \mathrm{B}$ activation, mostly activated by toll-like receptor 4 (TLR4)ligand lipopolysaccharide (LPS), promotes NLRP3 and pro-IL$1 \beta$ gene transcription; while the signal 2 triggers functional NLRP3 inflammasome activation by inflammasome ligands, including both pathogen-associated molecular patterns and endogenous damage associated molecular patterns (DAMPs). Among DAMP molecules, the NADPH oxidase (NOX)-derived reactive oxygen species (ROS) is a key factor that initiates the second signaling step (36).

Several studies indicated a close link between mir-21 and NLRP3 inflammasome. Ling et al. reported that mir-21 induces the ERK/NF- $\kappa \mathrm{B}$ pathway via degrading the target gene Spry1 (19). In addition, mir-21 increased ROS generation by decreasing the activity of superoxide dismutase (47). Furthermore, Smad2/3 mediated NOX4-derived ROS production (35). Hence, mir-21 has the potential to increase NOX4derived ROS production by targeting $S m a d 7$ for degradation. As a result, we infer that mir-21 activates the ERK/NF- $\kappa \mathrm{B}$, Smad/NOX4/ROS pathway via reducing Spry1 and Smad7, leading to activation of NLRP3 inflammasome/IL-1 $\beta$ axis.

Angiotensin II (AngII), a component of the reninangiotensin system (RAS), has been implicated in the pathogenesis of fibrosis in the kidneys (42), lungs (43), and liver (28). As a vasoconstrictive peptide, AngII triggers hypertension by activation and infiltration of certain subsets of immune cells $(11,16,40)$. In addition, being a pro-oxidative peptide, AngII triggers formation of NOX4-derived ROS (4, $25)$ and mitochondrial reactive oxygen species (mito-ROS) $(6,8,15)$, the mito-ROS lead to white blood cell activation and inflammation (17). Interestingly, there exists interaction and cross-activation between AngII-induced mito-ROS and NADPH oxidase-dependent $\operatorname{ROS}(6,8,15)$.

It is well known that the mito-ROS contribute to the activation of the NLRP3 inflammasome (50) providing the link between AngII-induced mito-ROS formation and inflammation. Our previous study (4) further confirmed that Ang II enhanced
NOX4-derived ROS production in HSCs, leading to NLRP3 inflammasome activation and resultant collagen synthesis.

Recent study reported that AngII promotes mir-21 expression (21), suggesting that mir-21 is likely to mediate AngIIinduced liver fibrosis. Nevertheless, the mechanism by which mir-21 mediates AngII-induced NLRP3 inflammasome activation and collagen synthesis in HSCs remains unclear.

Angiotensin-(1-7) [Ang-(1-7)], another peptide in RAS, regulates Ang II negatively (9). Ang-(1-7) is believed to protect against fibrosis in the liver (22) and lungs (25) by inhibiting the profibrotic effect of AngII. Reports regarding the relationship between Ang-(1-7) and mir-21 are rare and only one study has reported that Ang-(1-7) reduced mir-21 expression in the muscle (1). How mir-21 mediates the antifibrotic effects of Ang-(1-7) against hepatic fibrosis deserves further investigation.

Therefore, we hypothesized that AngII-induced mir-21 activates the ERK/NF- $\kappa \mathrm{B}, \mathrm{Smad} / \mathrm{NOX} 4 / \mathrm{ROS}$ pathway in HSCs via targeting Spry1 and Smad7, leading to NLRP3 inflammasome/IL- $1 \beta$ axis activation and thus contributing to liver fibrosis. Furthermore, we inferred that Ang-(1-7) inhibits AngII-induced mir-21 expression in liver fibrosis.

The present study aimed to investigate the role of AngIIinduced mir-21 in the regulation of NLRP3 inflammasome/IL$1 \beta$ axis in vitro and in vivo. We demonstrate that AngII-induced mir-2 1 activated NLRP3 inflammasome/IL-1 $\beta$ axis in HSCs via targeting Spryl and Smad7, resulting in HSC activation. Ang-(1-7) protected against bile duct ligation (BDL)- or AngII-induced liver fibrosis and inhibited mir-21 expression.

\section{Results}

\section{Circulating mir-21 level positively correlates with human liver fibrosis and oxidation}

We analyzed the correlation between mir-21 levels and human liver fibrosis. In situ hybridization (ISH) and quantitative reverse-transcription polymerase chain reaction (q-RTPCR) results showed that the ISHAK score/METAVIR score, mir-21 levels in blood and liver, liver FibroScan value, area density of Masson's trichrome staining, ALT/AST content, and $\mathrm{H}_{2} \mathrm{O}_{2}$ content from patients with liver fibrosis were significantly higher than those from the control group. The mir$21 \mathrm{ISH}, \alpha$-smooth actin ( $\alpha$-SMA), and von Willebrand factor (VWF) immunohistochemical (IHC) staining of serial section illustrated that the mir-21 was generated by HSCs. Association study showed a positive correlation of circulating mir-21 levels with liver mir-21 levels $(r=0.477, p=0.018)$, liver FibroScan value $(r=0.816, p=0.000)$, area density of Masson's trichrome staining $(r=0.785, p=0.000)$, ALT $(r=$ $0.606, p=0.002)$, AST $(r=0.575, p=0.003)$, and liver $\mathrm{H}_{2} \mathrm{O}_{2}$ content $(r=0.816, p=0.00)$ (Fig. 1).

\section{Upregulation of Smad2/3, NOX4, ERK, NF-кB, and NLRP3 inflammasome protein levels in human fibrotic liver tissue}

Smad7 and Spry1 protein levels in fibrotic liver tissue were lower than those in the control group. However, Smad2/3, NOX4, ERK, NF- $\kappa \mathrm{B}$, and NLRP3 inflammasome protein levels were higher in fibrotic liver tissue than in the control group (Supplementary Fig. S1; Supplementary Data are available online at www.liebertpub.com/ars). 
A
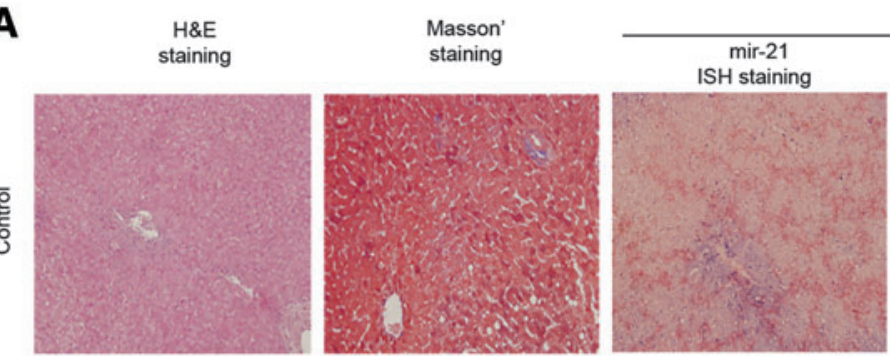

Serial section a-SMA
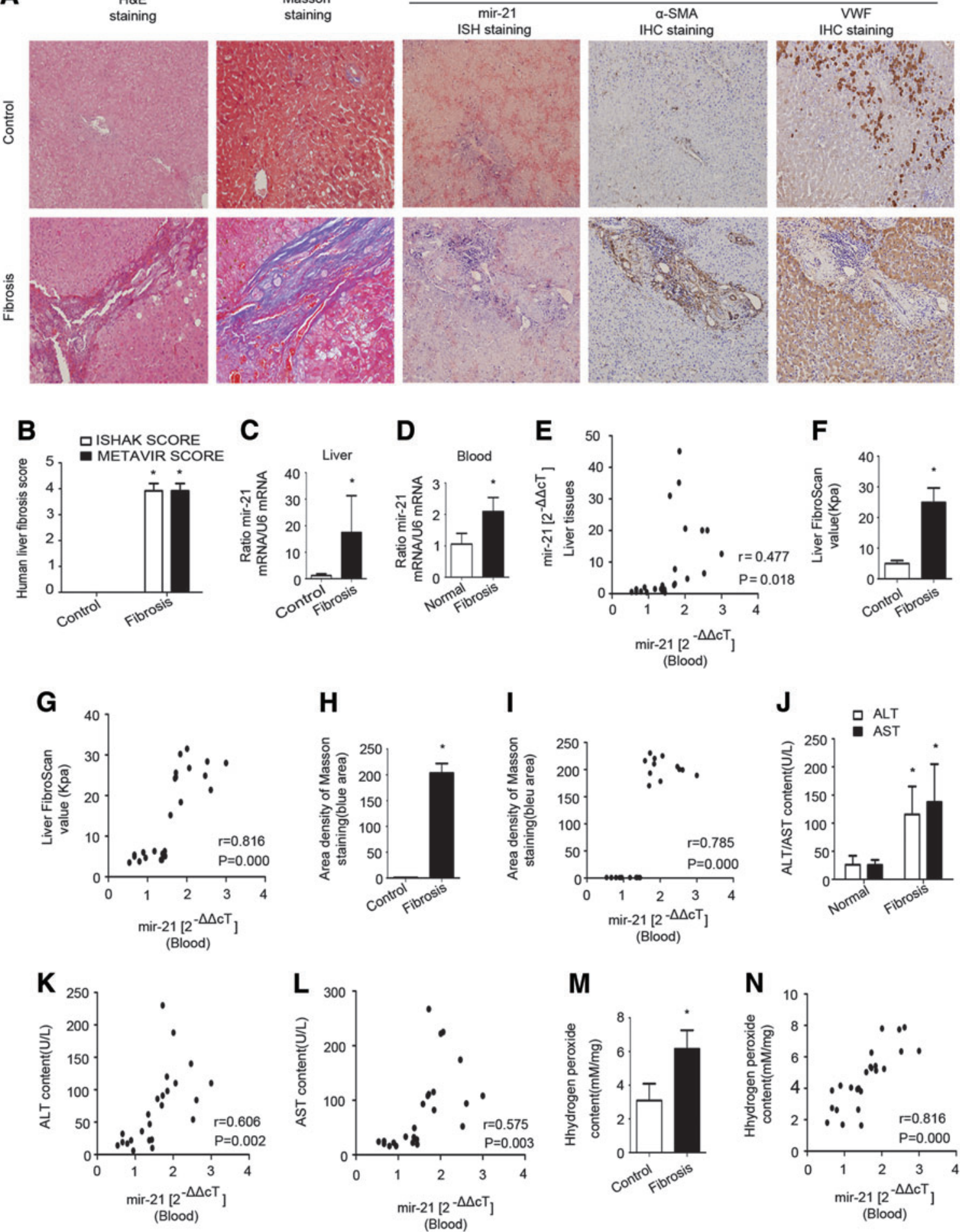

FIG. 1. Mir-21 correlates with human liver fibrosis and oxidation. (A) Representative pictures of H\&E and Masson's trichrome staining and mir-21 ISH and $\alpha$-SMA and VWF IHC staining of serial section. Original magnification: $\times 200$. (B) Morphological changes in fibrotic liver quantified using the ISHAK score and METAVIR score. (C-E) The levels of mir-21 in human liver tissues and blood were determined by q-RT-PCR. Correlation between blood mir-21 expression $\left(2^{-\Delta \Delta c t}\right)$ and liver tissue mir-2l expression $\left(2^{-\Delta \Delta c t}\right)$. (F, G) Instantaneous elasticity of normal and cirrhotic livers detected by FibroScan. Correlation between blood mir-21 expression $\left(2^{-\Delta \Delta \mathrm{ct}}\right)$ and liver FibroScan value (Kpa). (H, I) Quantitative measurement of area density of Masson's trichrome staining. Correlation between blood mir-2l expression $\left(2^{-\Delta \Delta \mathrm{ct}}\right)$ and area density of collagen. ( $\mathbf{J}-\mathbf{L})$ The contents of ALT and AST in serum of cirrhotic patients and normal persons. Correlation between blood mir-21 expression $\left(2^{-\Delta \Delta \mathrm{ct}}\right)$ and ALT/AST content $(\mathrm{U} / \mathrm{L})$. $(\mathbf{M}, \mathbf{N})$ The hydrogen peroxide content of liver tissue. Correlation between blood mir-21 expression $\left(2^{-\Delta \Delta \mathrm{ct}}\right)$ and hydrogen peroxide content. $n=12$ per group. The data are presented as mean \pm SEM. * $p<0.05$ versus the control or the normal. IHC, immunohistochemical; ISH, in situ hybridization; q-RT-PCR, quantitative reverse-transcription polymerase chain reaction; $\alpha$-SMA, $\alpha$-smooth actin; VWF, von Willebrand factor. To see this illustration in color, the reader is referred to the web version of this article at www.liebertpub.com/ars 
Ang-(1-7) infusion suppressed mir-21, Smad7/Smad2/3/NOX4, Spry1/ERK/NF-KB pathway, NLRP3 inflammasome, and liver fibrosis induced by $B D L$ or Angll infusion in rat liver

Ang-(1-7) treatment attenuated BDL or AngII infusioninduced liver fibrosis (Figs. 2A, C, D and 3A, C, D). The BDL- or AngII-treated rats were characterized by increased mir-21 expression. However, Ang-(1-7) reduced the expression of mir-21 (Figs. 2A, B and 3A, B). Moreover, BDL treatment and AngII infusion resulted in decreased Smad7 and Spry1 protein levels but increased Smad2/3/NOX4, ERK-NF- $\kappa$ B, NLRP3 inflammasome, $\alpha$-SMA, and $\alpha$ collagen I (COL1A) protein levels and $\mathrm{H}_{2} \mathrm{O}_{2}$ content in the liver, which was reversed by Ang-(1-7) infusion (Figs. 2A, E-H and $3 \mathrm{~A}, \mathrm{E}-\mathrm{G})$.

\section{Inhibition of mir-21 suppressed activation of Smad7/Smad2/3/NOX4, Spry1/ERK/NF-кB pathway, NLRP3 inflammasome, and liver fibrosis induced by Angll infusion}

Lenti-mir-21-down reduced liver fibrosis and the mir-21 expression induced by AngII (Fig. 4A-D). In addition, AngII infusion resulted in decreased $\mathrm{Smad} 7$ and Spry1 protein levels but increased Smad2/3/NOX4, ERK-NF- $\kappa$ B, NLRP3 inflammasome, $\alpha$-SMA, and COL1A protein levels and $\mathrm{H}_{2} \mathrm{O}_{2}$ content in the liver, which could be reversed by lenti-mir-21down treatment (Fig. 4A, E-G).

\section{Angll upregulated mir-21 level targeting Smad7 and Spry1 in HSCs}

AngII treatment increased mir-21 level (Fig. 5A, B). AngII-induced mir-21 expression was reduced by Smad3 siRNA but not by Smad2 siRNA (Fig. 5C, D), suggesting that Smad3 but not Smad2 mediates AngII-induced expression of mir-21 in HSCs. In addition, VAS2870, NAC, catalase, and mito-TEMPO inhibited the expression of mir-21 induced by AngII (Fig. 5E). Meanwhile, U0126 and BAY suppressed the expression of mir-21 induced by AngII (Fig. 5F). Computational predictions revealed two conserved targets of mir- 21 in the 3'UTR of Smad7 mRNA and one in the 3'UTR of Spryl mRNA (Fig. 5G, I). We constructed luciferase reporters containing these wild or mutated 3'UTR of Smad7 and Spryl binding sites for mir-21. Our results showed that overexpression of mir-21 significantly inhibited luciferase activity of the wild-type Smad7 and Spry1 3'UTR reporters but not the mutant-type Smad7 and Spryl 3'UTR reporters (Fig. 5H, J). The data indicated that Smad7 and Spryl are targets of mir-21.

\section{Overexpression of mir-21 promoted oxidation, mitochondrial dysfunction, and resultant HSC activation}

Overexpression of mir-21 increased $\mathrm{H}_{2} \mathrm{O}_{2}$ content, whereas downregulation of mir-21 decreased $\mathrm{H}_{2} \mathrm{O}_{2}$ content (Fig. 6A, B). SB431542, Smad3 inhibitor, inhibited the increase in $\mathrm{H}_{2} \mathrm{O}_{2}$ content induced by overexpression of mir-21, suggesting that $\mathrm{Smad} 3$ is required for ROS generation induced by mir-21 overexpression (Fig. 6C). Meanwhile, overexpression of mir-21 increased intracellular superoxide content, whereas downregulation of mir-21 decreased intracellular superoxide content (Fig. 6D, E). We further assessed the effect of mir-21 on mitochondrial function. The mitoROS was mildly increased by overexpression of mir- 21 but decreased by inhibition of mir- 21 expression. However, ATP levels changed in the opposite direction (Fig. 6F-I). $\alpha$-SMA and COL1A protein levels were increased by lenti-mir-21-up (Fig. 6J-N). VAS2870, NAC, and mito-TEMPO inhibited the increase in COL1A and $\alpha$-SMA protein levels induced by lenti-mir-21-up (Fig. 6L-N). Hence, overexpression of mir21 promotes oxidation, mitochondrial dysfunction, and resultant HSC activation.

\section{Ang-(1-7) suppressed mir-21 expression and oxidation induced by Angll}

Ang-(1-7) markedly decreased the expression of mir-21 and reduced mir-21 overexpression induced by AngII (Supplementary Fig. S2A-C). However, A779 reversed the effect of Ang-(1-7) (Supplementary Fig. S2C). Interestingly, AngII alone increased $\mathrm{H}_{2} \mathrm{O}_{2}$ content (Supplementary Fig. S2D). However, Ang-(1-7) and VAS2870 attenuated the increase in $\mathrm{H}_{2} \mathrm{O}_{2}$ content induced by AngII (Supplementary Fig. S2D). Meanwhile, A779 reversed the effect of Ang-(1-7) (Supplementary Fig. S2D).

\section{Overexpression of mir-21 activated NLRP3 inflammasome via the Spry1-ERK-NF-KB pathway in HSCS}

The protein levels of p-ERK, NLRP3 inflammasome complex (NLRP3, procaspase 1, and ASC), Pro-IL-1 $\beta$, and IL-1 $\beta$ (P17) were increased by lenti-mir-21-up (Fig. 7A, B). However, overexpression of the Spryl plasmid reversed the effect of lenti-mir-21-up (Fig. 7A, B). Both U0126 and BAY inhibited NF- $\kappa \mathrm{B}$ translocation and the protein levels of ProIL-1 $\beta$, P17, NLRP3 inflammasome complex, and caspase 1(P10) induced by lenti-mir-21-up (Fig. 7C-F). These data demonstrate that mir-21 activated the NLRP3 inflammasome/IL- $1 \beta$ axis via the Spry1-ERK-NF- $\kappa$ B pathway.

\section{Overexpression of mir-21 promoted NLRP3 \\ inflammasome complex assembly in the mitochondria}

Immunofluorescence colocalization analysis was used to examine the localization of ASC, NLRP3, or caspase- 1 in the mitochondria. The data demonstrated that mir-21 overexpression promoted NLRP3 inflammasome to assemble in mitochondria. Furthermore, mir- 21 overexpression promoted NOX4 to colocalize with NLRP3, indicating that NOX4 reacted with NLRP3 in the mitochondria (Fig. 8A-D).

\section{Overexpression of mir-21 promoted NLRP3 \\ inflammasome activation and resultant collagen production via the Smad7/Smad2/3/NOX4 pathway in HSCs}

P-smad2/3, NOX4, NLRP3 inflammasome complex, P17, COL1A, and $\alpha$-SMA protein levels were increased by lentimir-21-up (Fig. 9A). However, overexpression of Smad7 plasmid reversed the effect of lenti-mir-21-up (Fig. 9A). Moreover, SB431542 reduced the increase in NOX4, NLRP3 
A
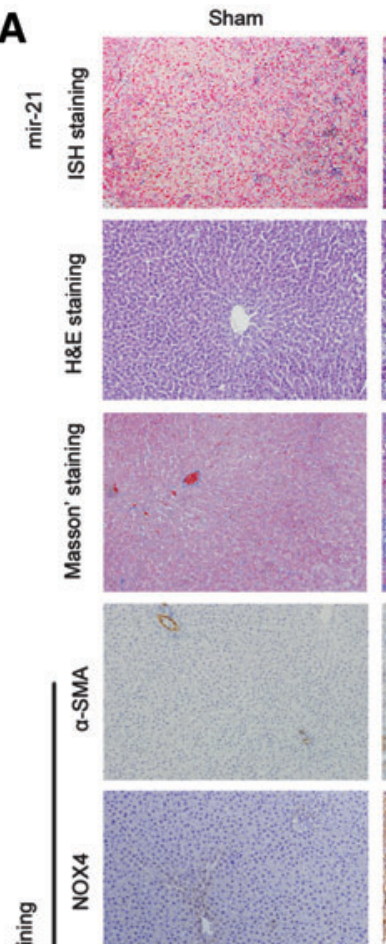

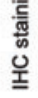

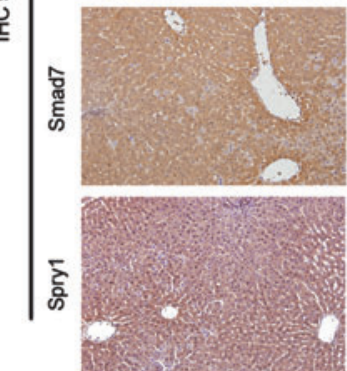

F

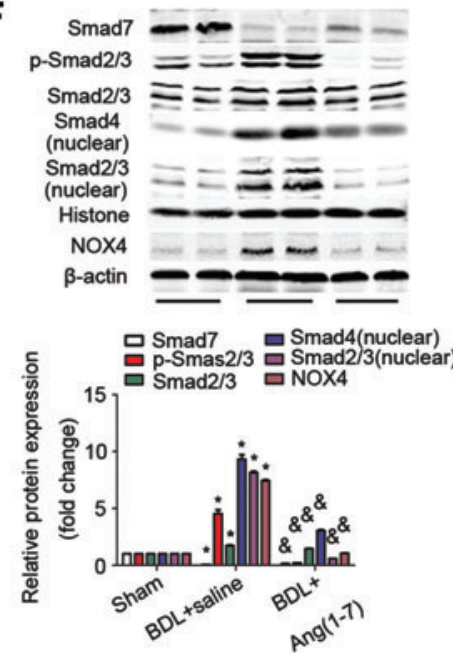

$B D L+$ saline
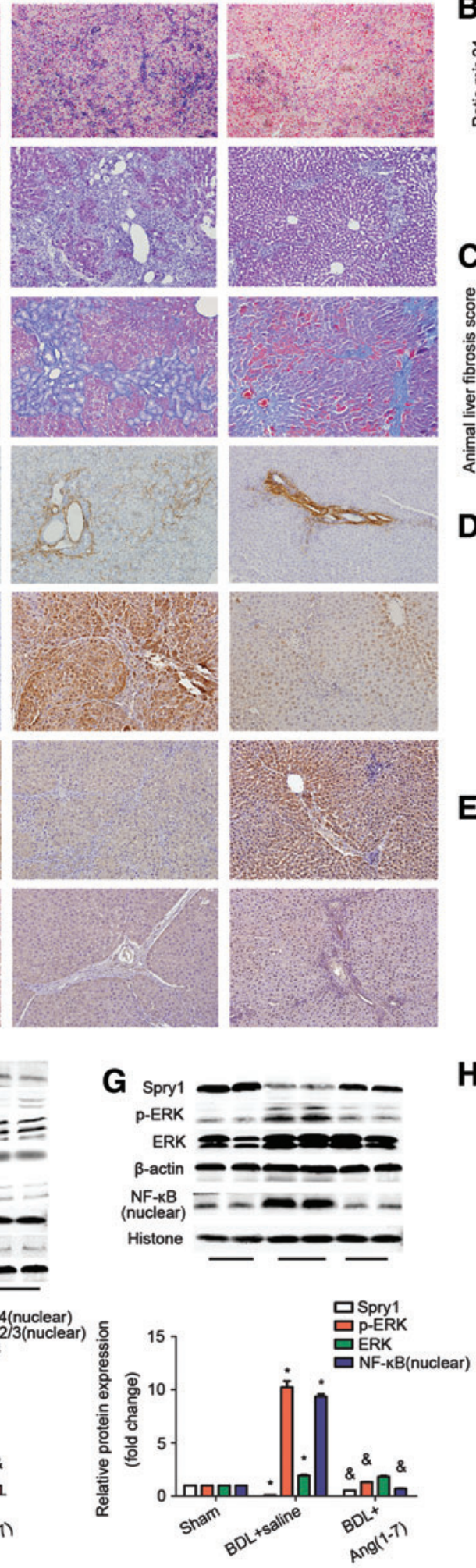

E
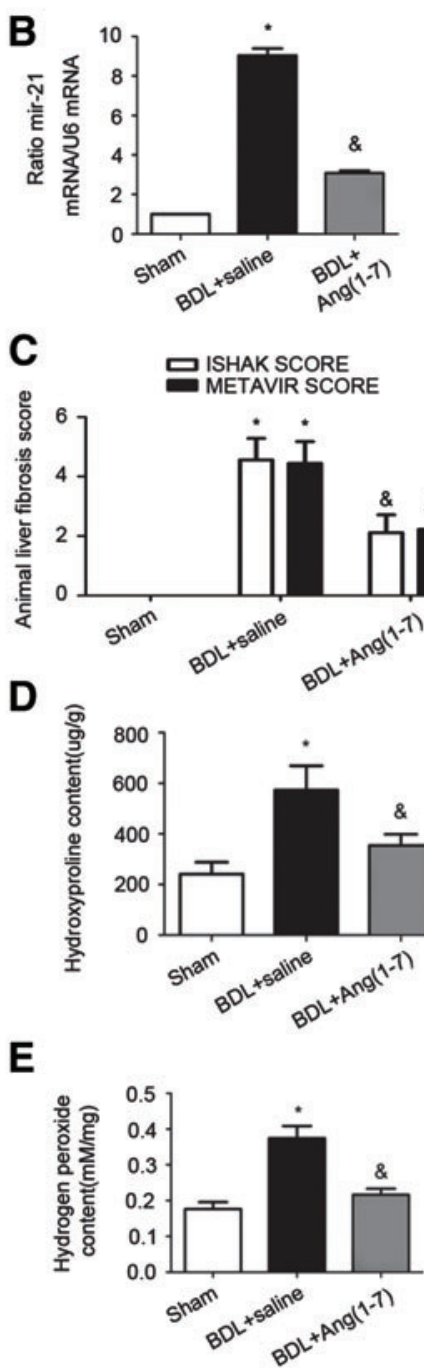

H
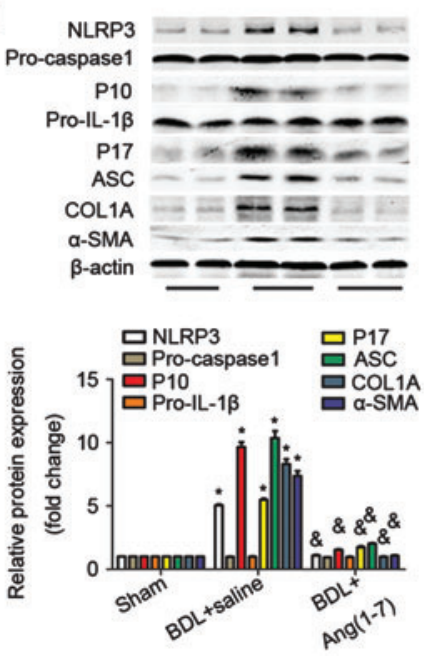

FIG. 2. Ang(1-7) infusion suppressed mir-21, Smad7/Smad2/3/NOX4, Spry1/ERK/NF- $\kappa$ B pathway, NLRP3 inflammasome, and liver fibrosis after BDL in the rat liver. (A) Representative pictures of liver sections stained with H\&E and Masson's trichrome. IHC staining was performed to determine the localization and expression of $\alpha$-SMA, NOX4, Smad7, and Spry 1 proteins. ISH staining was performed to determine the localization and expression of mir-21. Original magnification: $\times 200$. (B) Expression of mir-21 in the liver was determined by q-RT-PCR. (C) Morphological changes in fibrotic liver quantified using the ISHAK and METAVIR scores. (D, E) The hydroxyproline and hydrogen peroxide content in the liver in different groups. (F-H) Smad7, p-Smad2/ 3 , Smad4, Smad2/3, NOX4, Spry1, p-ERK, NF- $\kappa$ B, NLRP3, caspase1, ASC, IL-1 $\beta, \alpha$-SMA, and COL1A protein levels in liver tissues were analyzed by Western blotting. $n=9$ per group. The data are presented as mean \pm SEM. ${ }^{*} p<0.05$ versus the Sham group; ${ }^{*} p<0.05$ versus the BDL group. ASC, apoptosis-associated speck-like protein containing a caspase-recruitment domain; BDL, bile duct ligation; NLRP3, NOD-like receptor family, pyrin domain containing 3; NOX, NADPH oxidase. To see this illustration in color, the reader is referred to the web version of this article at www.liebertpub.com/ars 
A
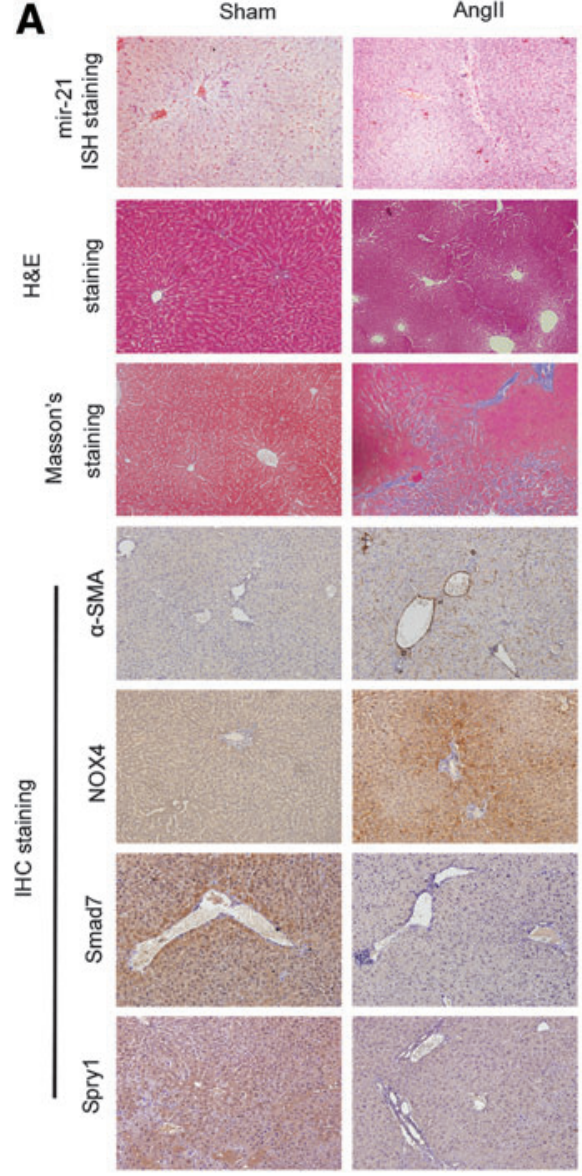

F
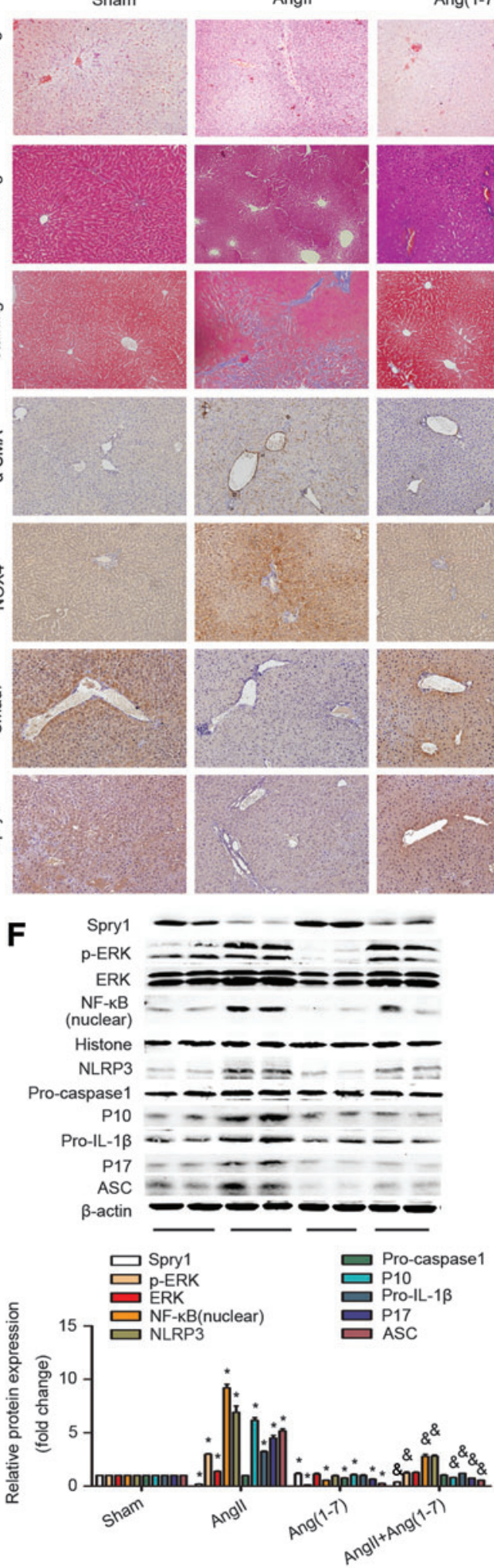

Ang(1-7)
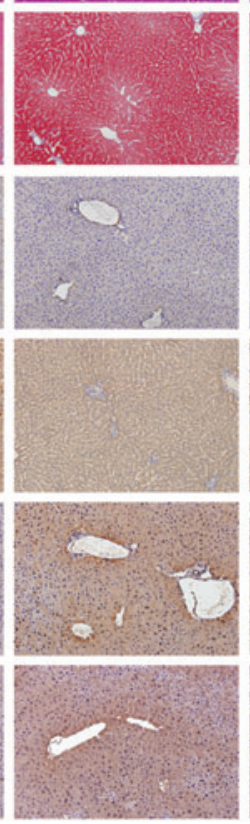

\section{G}
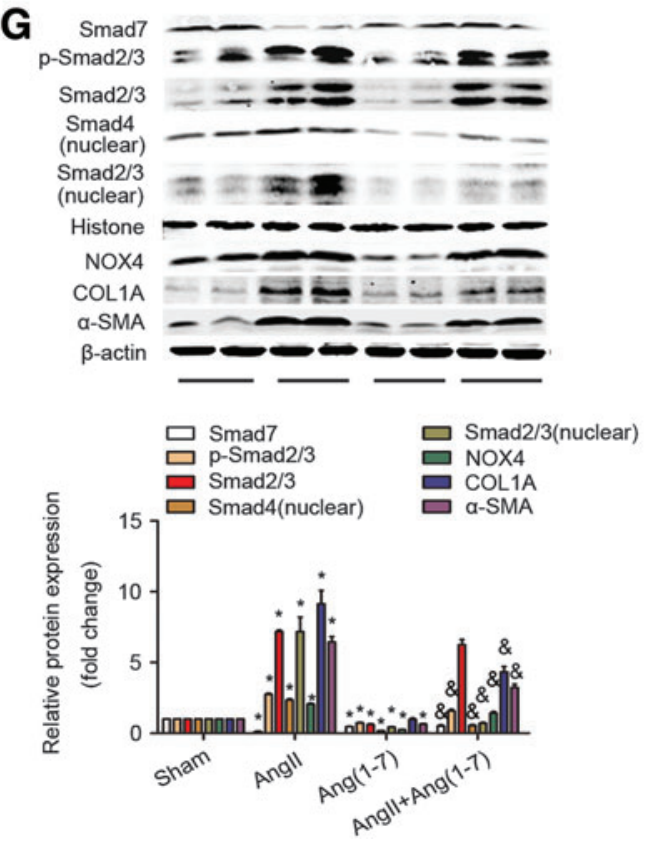

FIG. 3. Ang(1-7) infusion suppressed mir-21, Smad7/Smad2/3/NOX4, Spry1/ERK/NF- $\kappa$ B pathway, NLRP3 inflammasome, and liver fibrosis induced by AngII infusion in rat liver. (A) Representative pictures and quantitative measurement of liver sections stained with H\&E and Masson's trichrome. IHC staining was performed to determine the localization and expression of $\alpha$ SMA, NOX4, Smad7, and Spry1 proteins. ISH staining was performed to determine the localization and expression of mir-21. Original magnification: $\times 200$. (B) The expression of mir-21 in livers was determined by RT-qPCR. (C) Morphological changes in fibrotic liver quantified using the ISHAK and METAVIR scores. (D, E) The hydroxyproline and hydrogen peroxide content of livers in different groups. (F, G) The protein levels of Smad7, p-Smad2/3, Smad4, Smad2/3, NOX4, Spry1, p-ERK, NF- $\kappa$ B, NLRP 3 inflammasome, $\alpha$-SMA, and COL1A in liver tissues were analyzed by Western blot. $n=9$ per group. The data are presented as the mean \pm SEM. $* p<0.05$ versus the Sham group; ${ }^{*} p<0.05$ versus the AngII treatment group. AngII, angiotensin II. To see this illustration in color, the reader is referred to the web version of this article at www.liebertpub.com/ars 
A
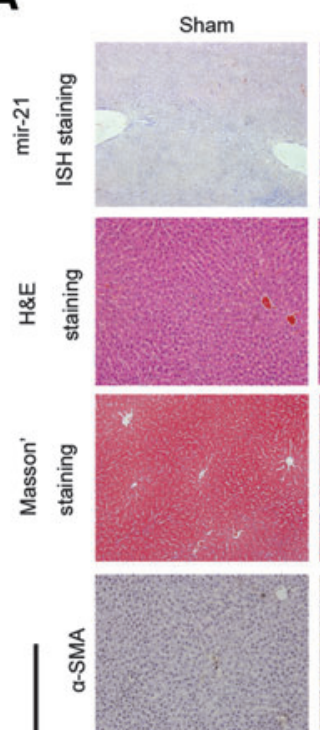

突
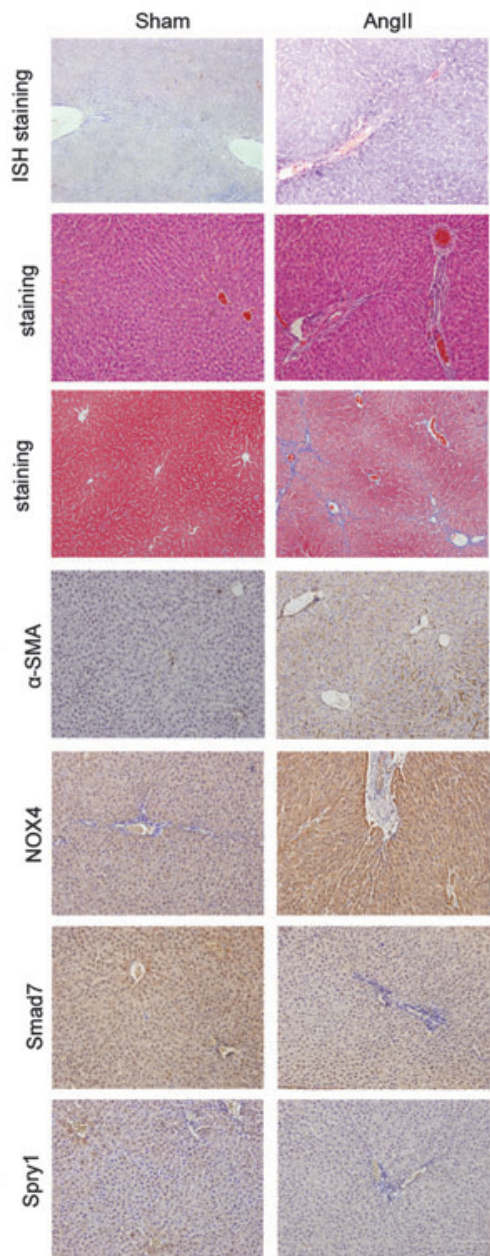

$\boldsymbol{F}_{\mathrm{p} \text {-ERK }}^{\mathrm{SpR} 1}= \pm$ ERK$$
\begin{gathered}
\mathrm{NF}-\mathrm{KB} \\
\text { (nuclear) }
\end{gathered}
$$

(nuclear)

NLRP3

Pro-caspase 1

P10

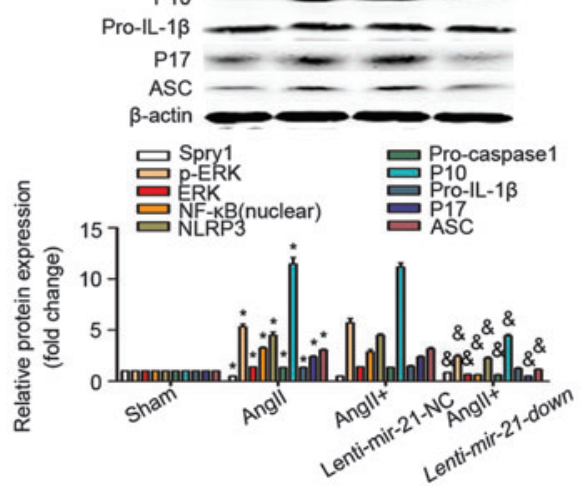

Angll+
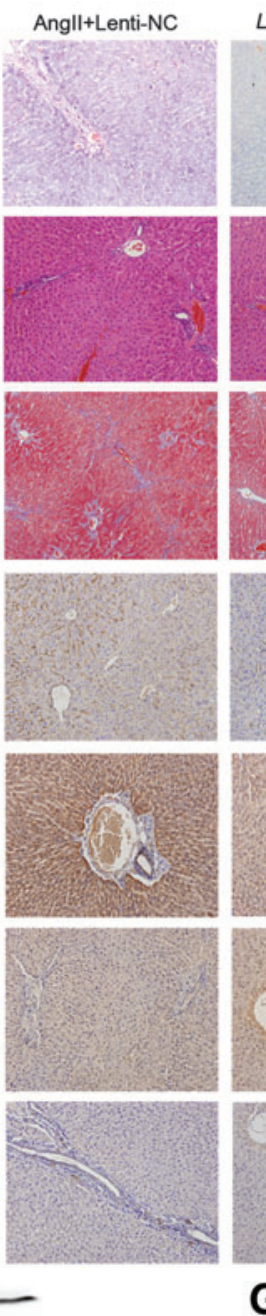

G
B
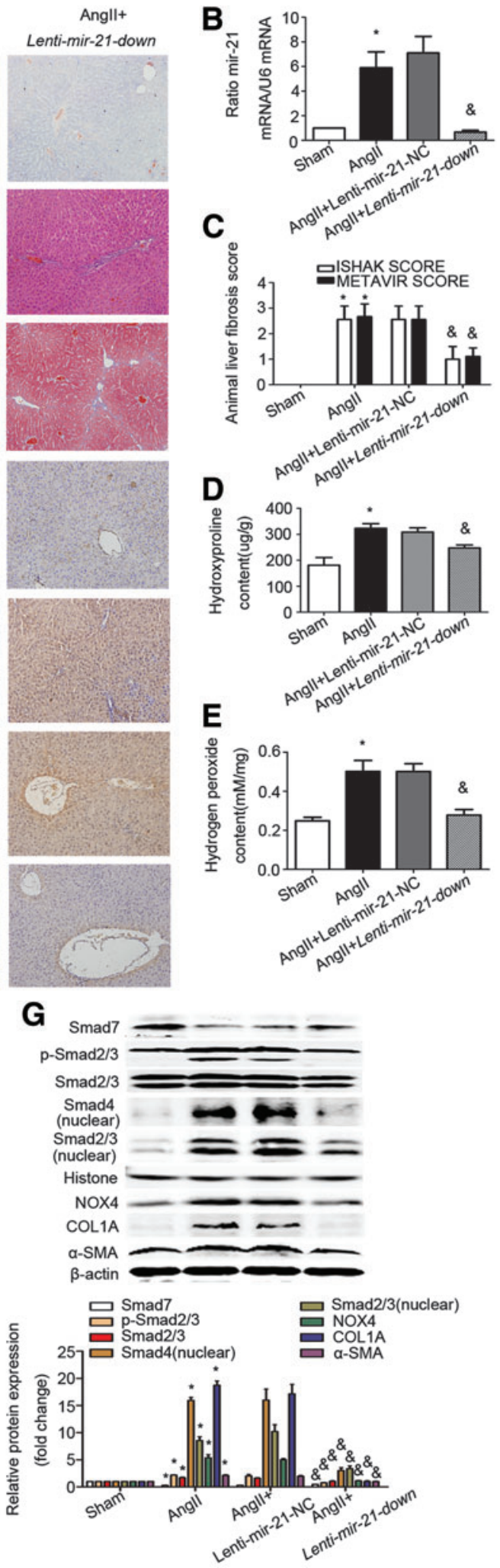

FIG. 4. Inhibition of mir-21 suppressed Smad7/Smad2/3/NOX4, Spry1/ERK/NF- $\kappa$ B pathway, NLRP3 inflammasome, and liver fibrosis induced by AngII infusion. (A) Representative pictures and quantitative measurement of liver sections stained with H\&E and Masson's trichrome. IHC staining was performed to determine the localization and expression of $\alpha$-SMA, NOX4, Smad7, and Spry1 proteins. ISH staining was performed to determine the localization and expression of mir-21. Original magnification: $\times 200$. (B) Mir-21 expression in the liver was determined by RT-qPCR. (C) Morphological changes in the fibrotic liver quantified using the ISHAK and METAVIR scores. (D, E) The hydroxyproline and hydrogen peroxide content in the different groups. (F, G) Smad7, p-Smad2/3, Smad4, Smad2/3, NOX4, Spry1, p-ERK, NF- $\kappa$ B, NLRP3, caspase1, ASC, IL-1 $\beta, \alpha$-SMA, and COL1A protein levels in liver tissues were analyzed by Western blotting. The data are presented as mean \pm SEM. ${ }^{*} p<0.05$ versus the Sham group; ${ }^{*} p<0.05$ versus the AngII treatment group. To see this illustration in color, the reader is referred to the web version of this article at www.liebertpub.com/ars 
NING ET AL.
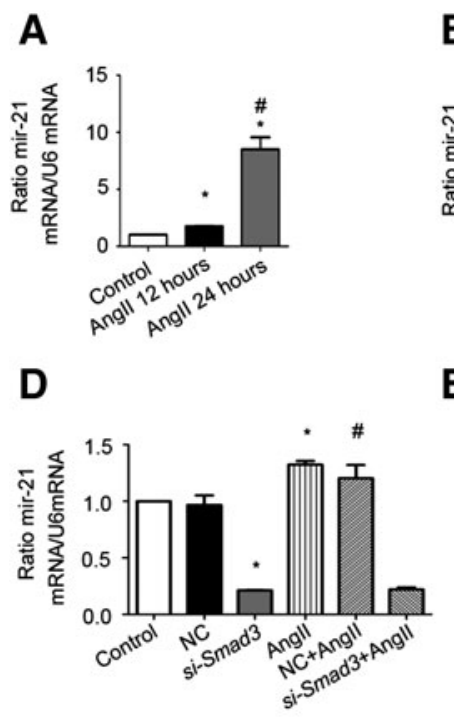

B

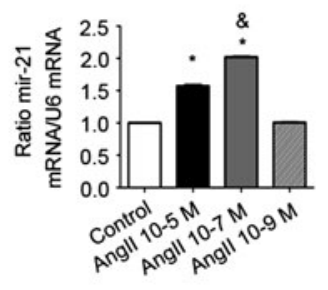

E

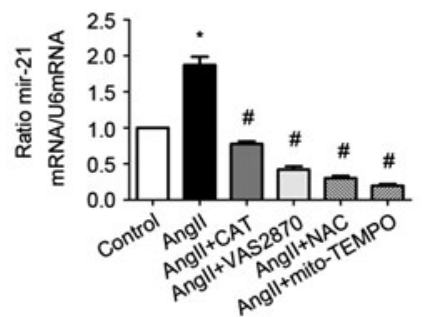

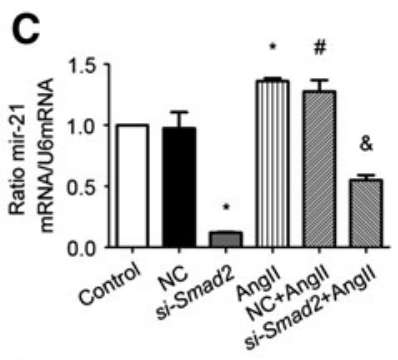

F

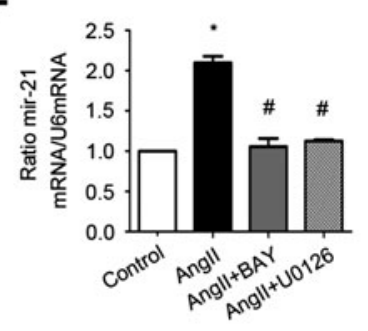

G

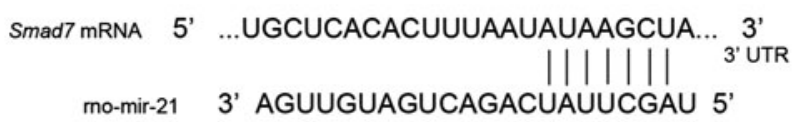

H

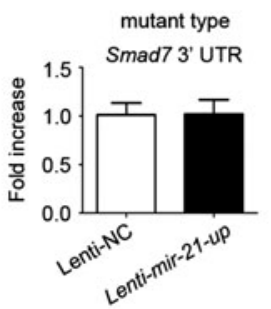

I

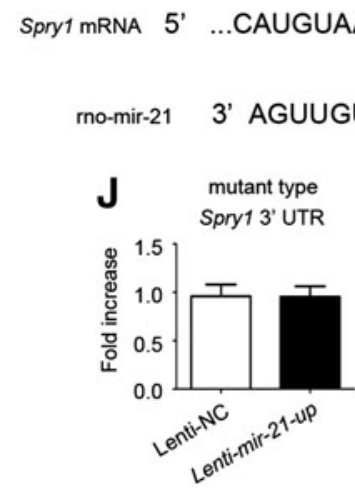

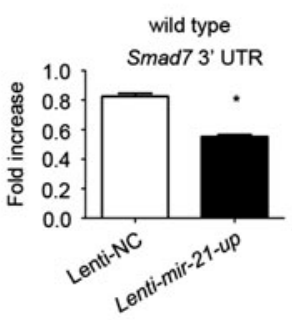

wild type 

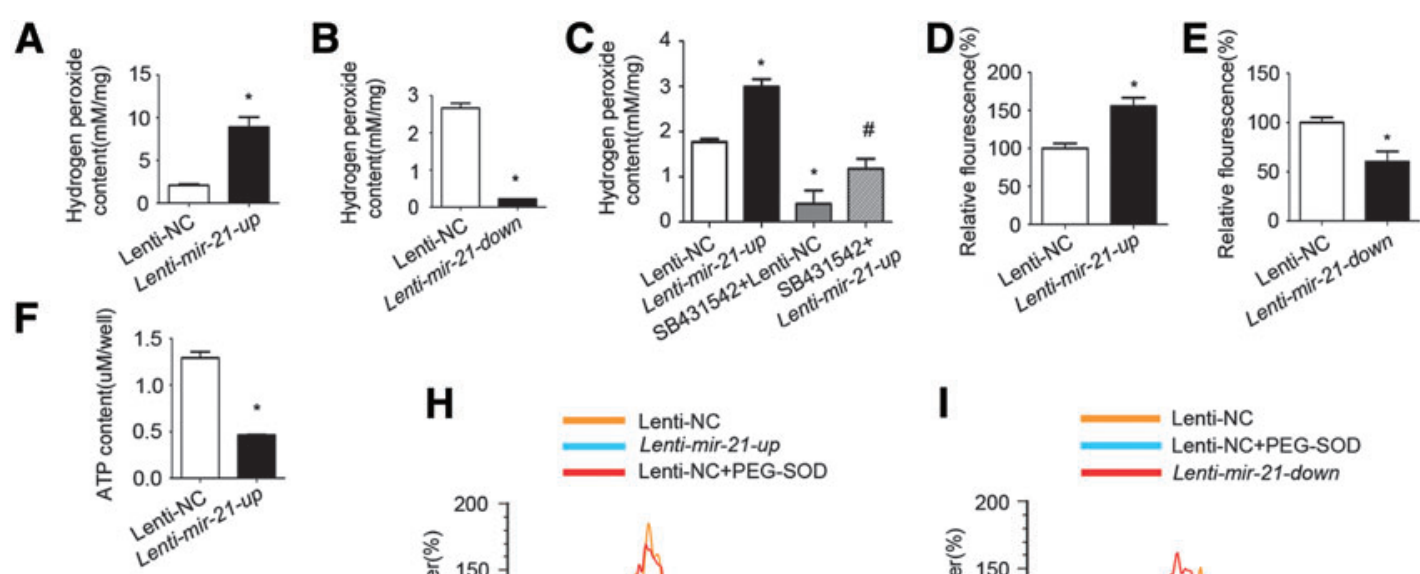

G
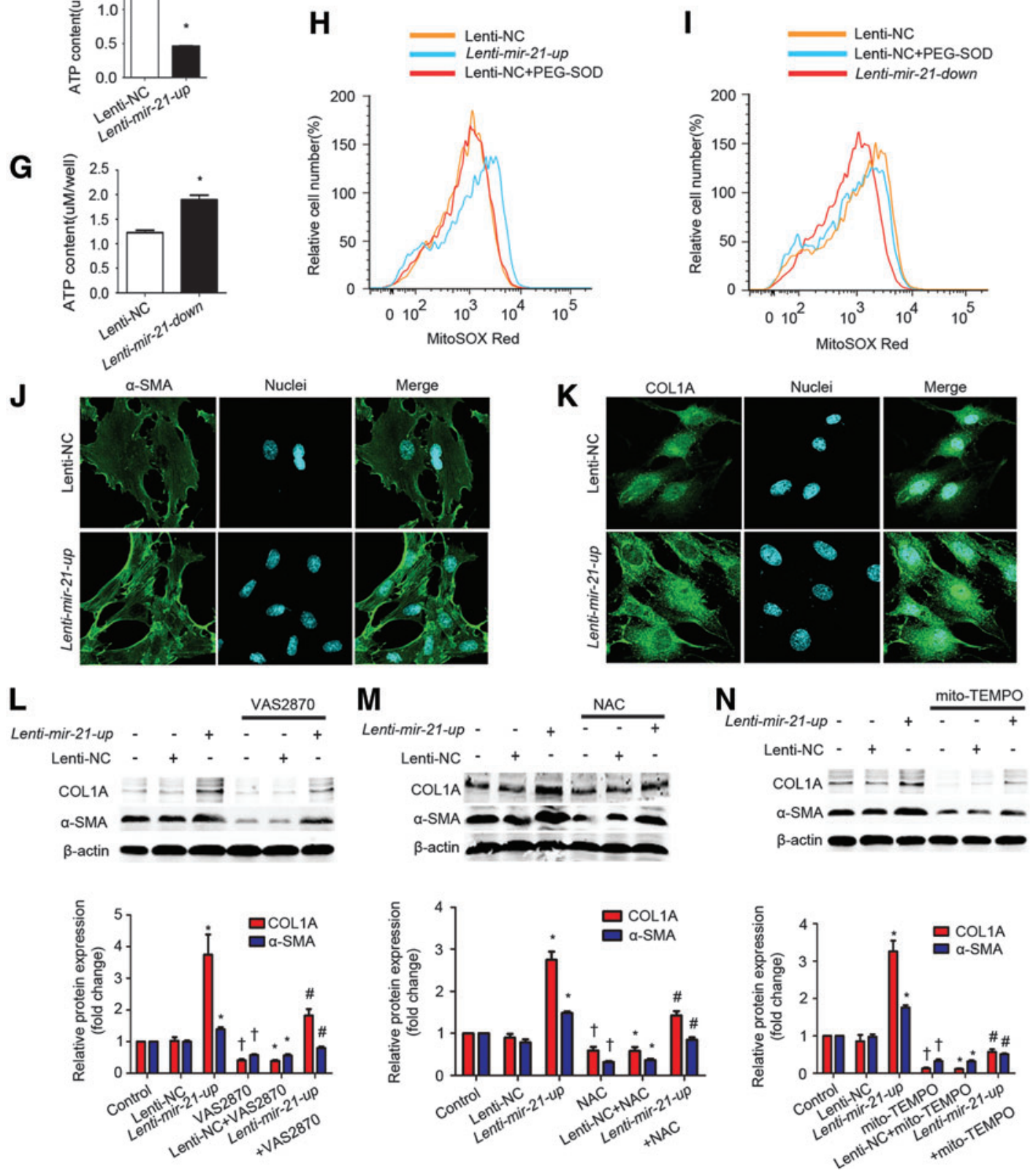

FIG. 6. Overexpression of mir-21 promoted oxidation, mitochondrial dysfunction, and resultant HSC activation. HSCs were transfected with lenti-mir-21-up or lenti-mir-21-down. (A, B) The $\mathrm{H}_{2} \mathrm{O}_{2}$ concentration in HSCs was measured. The data are presented as the mean \pm SEM. ${ }^{*} p<0.05$ versus lenti-mir-21-NC. (C) HSCs were treated with SB431542 (50 $\left.\mu M\right)$ and transfected with lenti-mir21-NC or lenti-mir-21-up and $\mathrm{H}_{2} \mathrm{O}_{2}$ concentration in HSCs was measured. The data are presented as the mean \pm SEM. ${ }^{*} p<0.05$ versus lenti-mir-21-NC; ${ }_{p} p<0.05$ versus lenti-mir-21-up or SB431542+lenti-mir-21-NC. (D, E) The intracellular superoxide in HSCs was measured. The data are presented as the mean \pm SEM. * $p<0.05$ versus lenti-mir-21-NC. (F, G) ATP levels were measured in HSCs. The data are presented as the mean \pm SEM. ${ }^{*} p<0.05$ versus lenti-mir-21-NC. (H, I) HSCs transfected with lenti-NC were treated with PEG-SOD, mitochondria-derived reactive oxygen species were measured by flow cytometry. $(\mathbf{J}, \mathbf{K})$ Representative confocal immunofluorescence images of COL1A or $\alpha$-SMA. (L-N) HSCs were treated with VAS2870 $(50 \mu M)$, NAC $\left(10^{-3} M\right)$, mito-TEMPO (100 U/ml), and transfected with lenti-mir-2l-NC or lenti-mir-2l-up. Western blotting was used to detect COL1A and $\alpha$-SMA protein levels. The data are presented as mean \pm SEM. ${ }^{*} p<0.05$ versus lenti-mir-21-NC; ${ }^{p} p<0.05$ versus control; ${ }^{\#} p<0.05$ versus lenti-mir-21-up. All of the assays were performed in triplicate. PEG-SOD, polyethylene glycol-adsorbed-superoxide dismutase. To see this illustration in color, the reader is referred to the web version of this article at www.liebertpub.com/ars. 

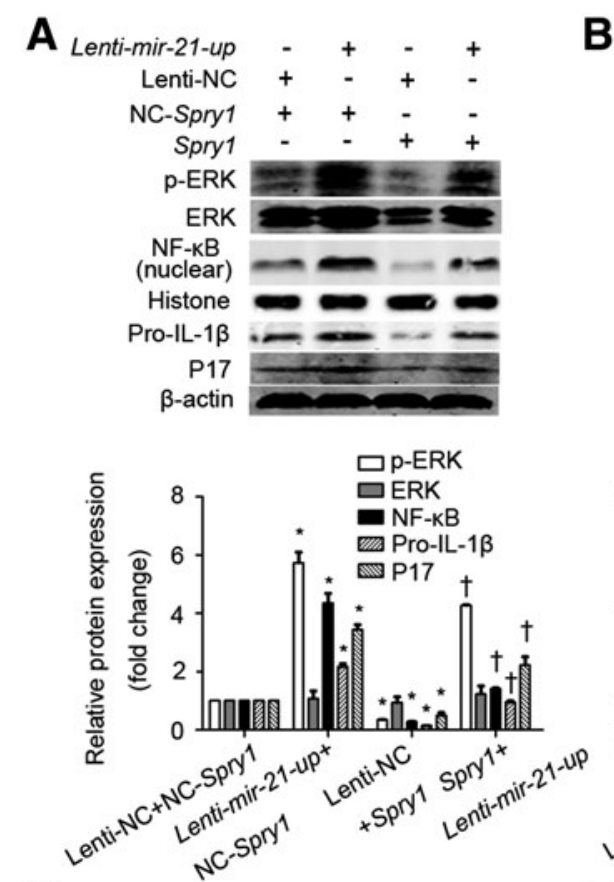

B
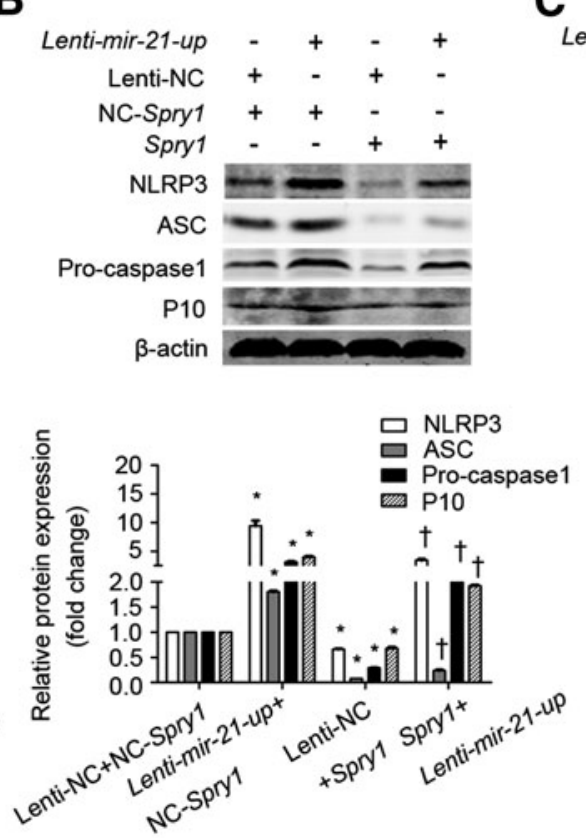
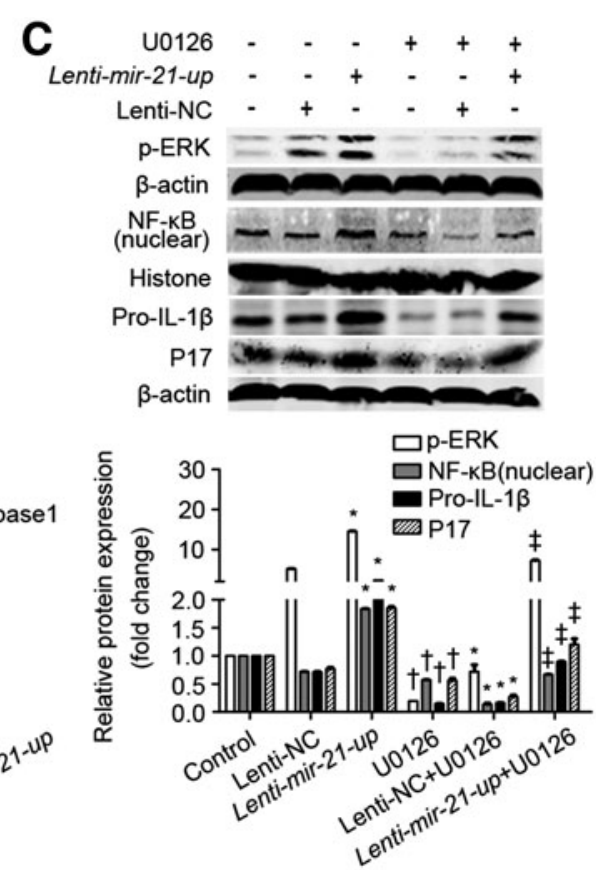
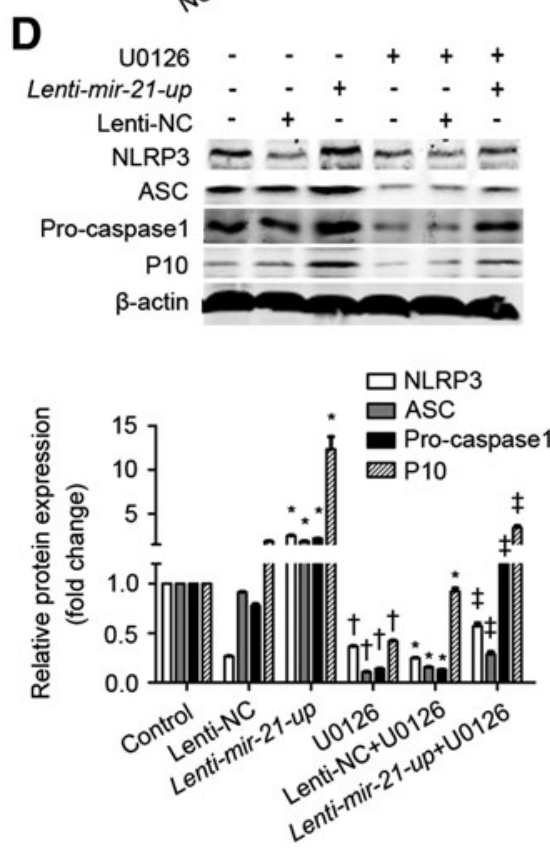

$E$
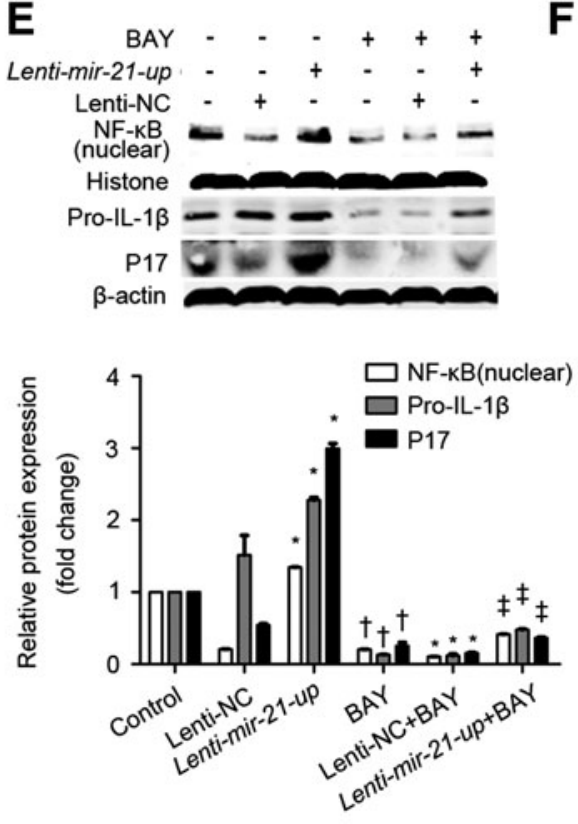

$\mathbf{F}$

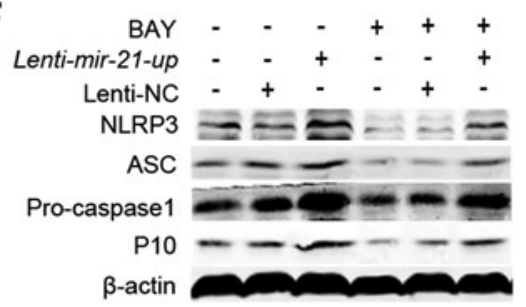

FIG. 7. Overexpression of mir-21 activated NLRP3 inflammasome via the Spry1-ERK-NF- $\kappa$ B pathway in HSCs. HSCs were transfected with Spryl overexpression plasmid and lenti-mir-21-NC or lenti-mir-21-up. (A, B) p-ERK, Pro-IL-1 $\beta$, P17, NLRP3, ASC, procaspase1, and P10 protein levels were analyzed by Western blotting. The data are presented as the mean \pm SEM. $* p<0.05$ versus lenti-NC+NC-Spry1; ${ }^{\dagger} p<0.05$ versus lenti-mir- 21 -up $+\mathrm{NC}-$ Spryl. (C, D) HSCs were treated with U0126 $\left(10^{-5} M\right)$ and transfected with lenti-mir-21-NC or lenti-mir-21-up. p-ERK, NF- $\kappa$ B, Pro-IL$1 \beta$, P17, NLRP3, ASC, procaspase1, and P10 protein levels were analyzed by Western blot. (E, F) HSCs were treated with BAY $\left(10^{-5} M\right)$ and transfected with lenti-mir-21-NC or lenti-mir-21-up. NF- $\kappa$ B, Pro-IL-1 $\beta$, P17, NLRP3, ASC, procaspase1, and P10 protein levels were analyzed by Western blot. The data are presented as mean \pm SEM. $* p<0.05$ versus lenti-mir- 21 -NC; ${ }^{\dagger} p<0.05$ versus control; ${ }^{\star} p<0.05$ versus lenti-mir-21-up. All of the assays were performed in triplicate.

inflammasome complex, P17, COL1A, and $\alpha$-SMA protein levels induced by lenti-mir-21-up (Fig. 9B). On the contrary, lenti-mir-21-up resulted in increased protein level of NOX4, other than NOX2. VAS2870, NAC, and mito-TEMPO attenuated the increase in NLRP3 inflammasome complex and P17 protein levels induced by lenti-mir-21-up (Fig. 9C-E).
Collectively, these data suggest that mir-21 activated the NLRP3 inflammasome/IL-1 $\beta$ axis by the Smad7/Smad2/3/ NOX4 pathway in HSCs. Furthermore, NLRP3 siRNA significantly reduced the mir-21 overexpression-induced COL1A and $\alpha$-SMA production, suggesting that NLRP3 mediates HSC activation and collagen synthesis (Fig. 9F). 

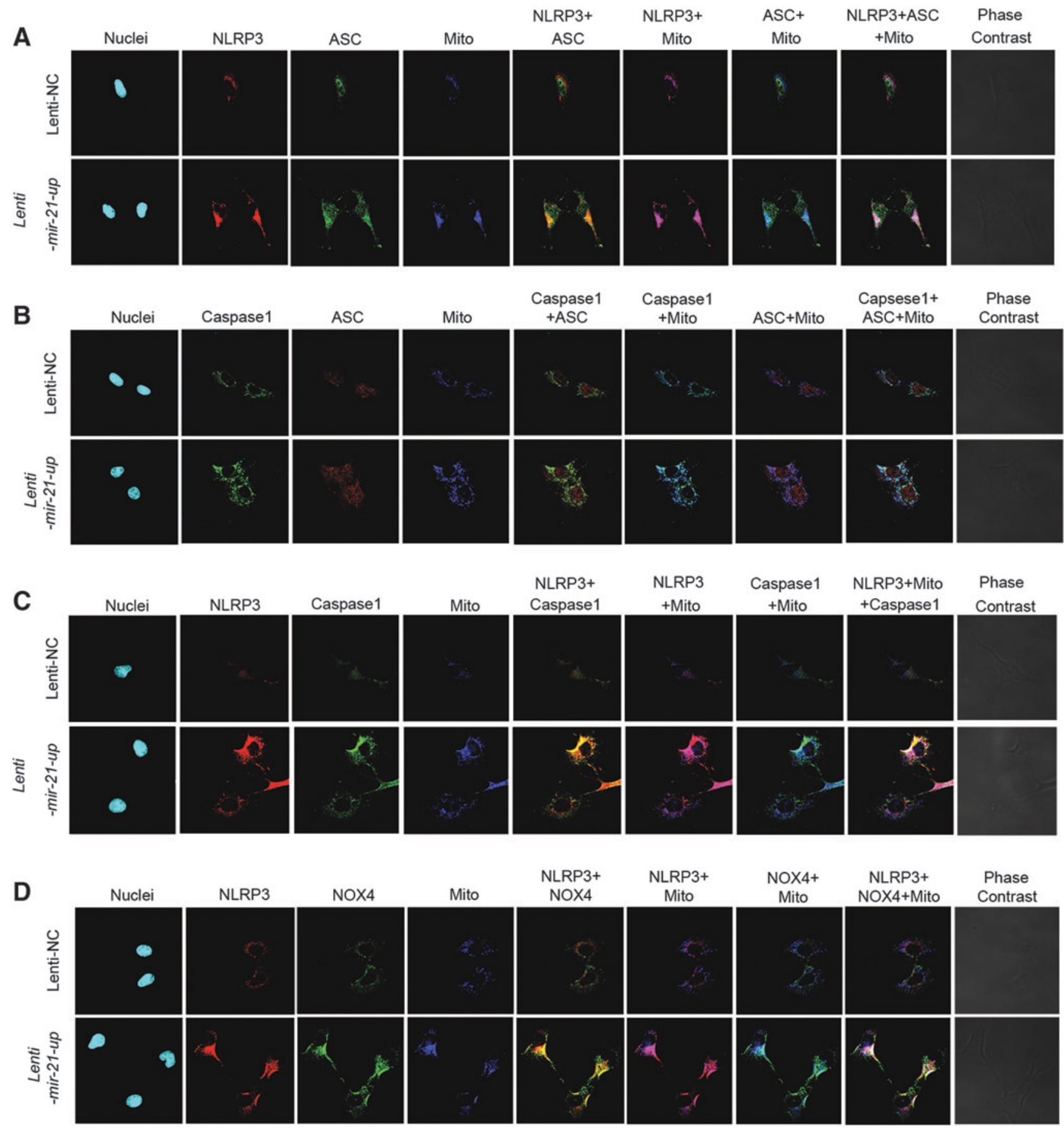

Phase Contrast

FIG. 8. Overexpression of mir-21 promoted the assembly of NLRP3 inflammasome components in mitochondria in HSCs. Cells were transfected with lenti-mir-21-NC or lenti-mir-21-up and then were used to perform immunofluorescence staining. Nuclei were stained with DAPI; mitochondria were stained with mito-tracker (blue). (A) Representative confocal immunofluorescence images of NLRP3 (red) colocalized with ASC (green) and mitochondria (blue). (B) Representative confocal immunofluorescence images of caspase-1 (green) colocalized with ASC (red) and mitochondria (blue). (C) Representative confocal immunofluorescence images of NLRP3 (red) colocalized with caspase1 (green) and mitochondria (blue). (D) Representative confocal immunofluorescence images of NLRP3 (red) colocalized with NOX4 (green) and mitochondria (blue). Original magnification: $\times 1200$. All of the staining was performed in triplicate. To see this illustration in color, the reader is referred to the web version of this article at www.liebertpub.com/ars 
A
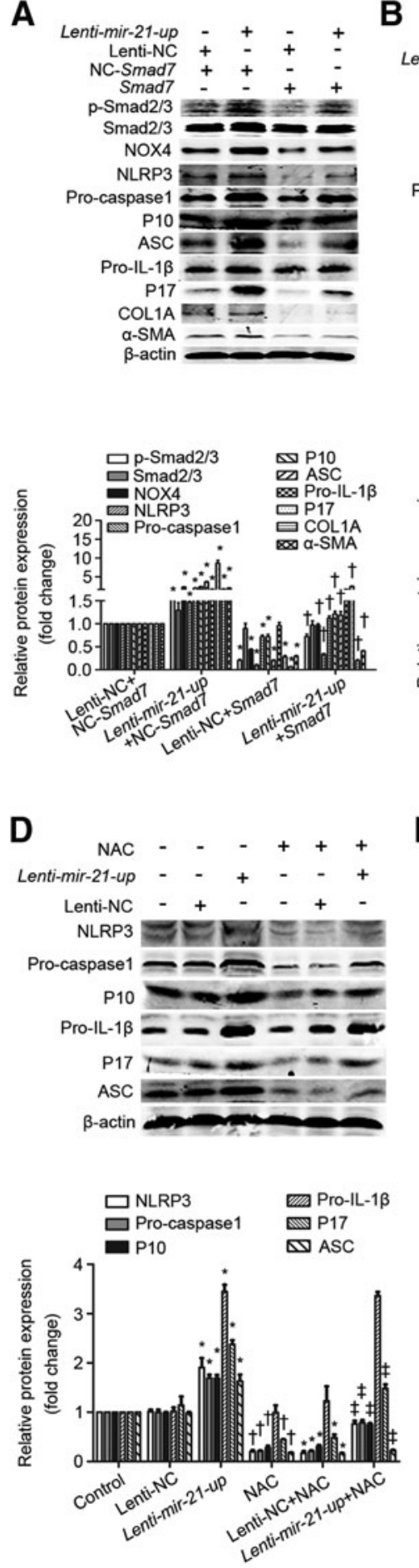

B
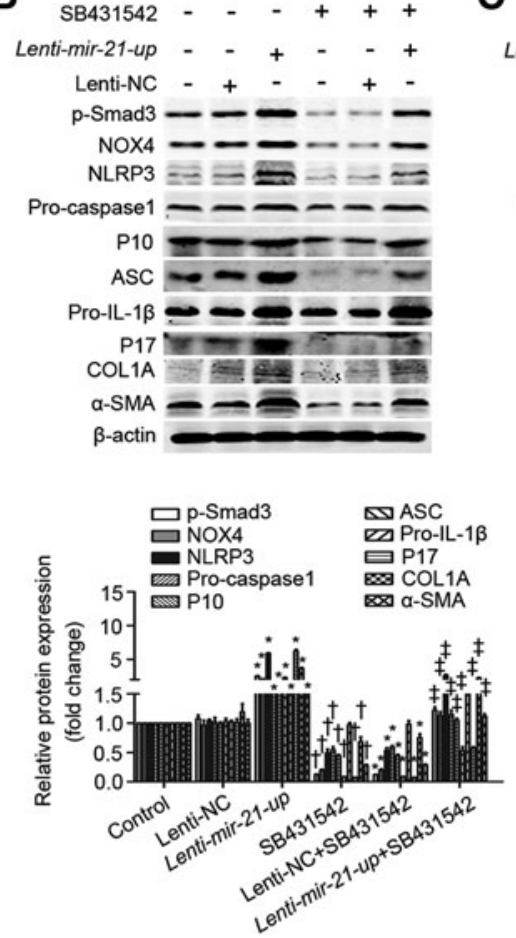

E
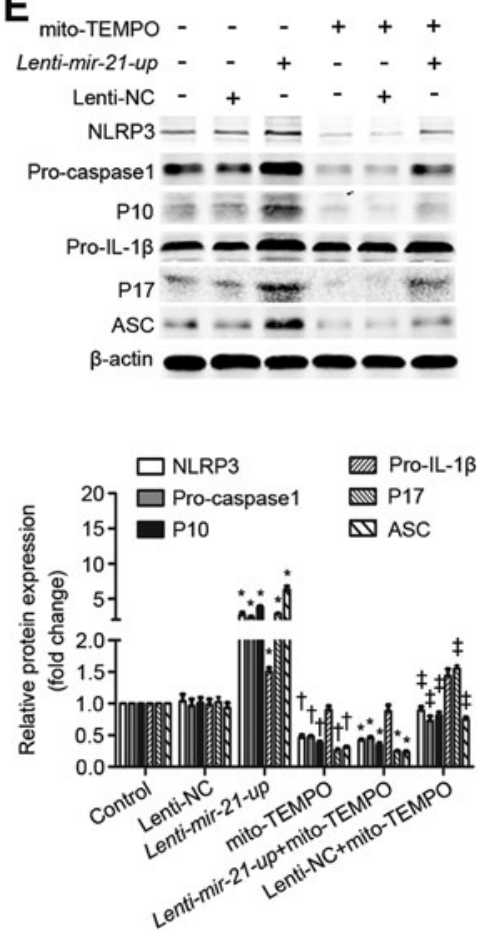

C
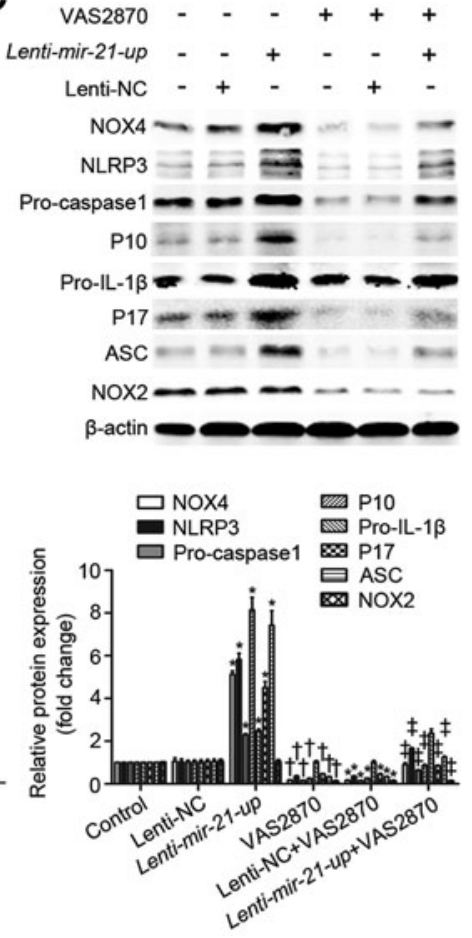

$\mathbf{F}$
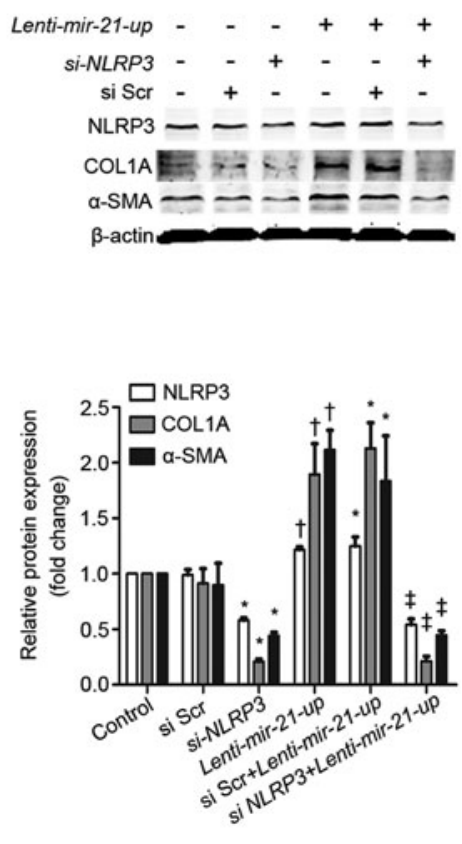

FIG. 9. Overexpression of mir-21 promoted NLRP3 inflammasome activation and resultant collagen production via the Smad7/Smad2/3/NOX4 pathway in HSCs. (A) HSCs were transfected with Smad7 overexpression plasmid and lentimir-21-NC or lenti-mir-21-up. p-Smad2/3, NOX4, NLRP3, ASC, procaspase1, P10, P17, $\alpha$-SMA, and COL1A protein levels were analyzed by Western blot. The data are presented as mean \pm SEM. ${ }^{*} p<0.05$ versus lenti-NC+NC-Smad7; ${ }^{\dagger} p<0.05$ versus lenti-mir-21-up+NC-Smad7. (B) HSCs were treated with SB431542 $(50 \mu M)$ and transfected with lenti-mir21-NC and lenti-mir-21-up. p-Smad3, NOX4, NLRP3, ASC, procaspase1, P10, P17, $\alpha$-SMA, and COL1A protein levels were analyzed by Western blot. (C-E) HSCs were treated with VAS2870 $(50 \mu M)$, NAC $\left(10^{-3} M\right)$, and mito-TEMPO $(100 \mathrm{U} / \mathrm{ml})$ and transfected with lenti-mir-21-NC or lenti-mir-21-up. NOX4, NOX2, NLRP3, ASC, procaspase1, P10, and P17 protein levels were analyzed by Western blot. The data are presented as the mean \pm SEM. * $p<0.05$ versus lenti-mir-21$\mathrm{NC} ;{ }^{\dagger} p<0.05$ versus control; ${ }^{\circledR} p<0.05$ versus lenti-mir-21-up. (F) HSCs were cotransfected with NLRP3 siRNA, lenti-mir21-NC, or lenti-mir-21-up. NLRP3, $\alpha$-SMA, and COL1A protein levels were analyzed by Western blot. The data are presented as mean \pm SEM. $* p<0.05$ versus si Scr; ${ }^{\dagger} p<0.05$ versus control; ${ }^{\star} p<0.05$ versus si Scr+lenti-mir-21-up. All of the assays were performed in triplicate. 
A

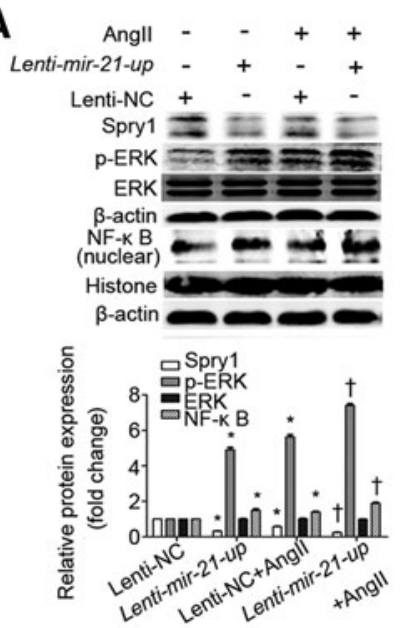

D
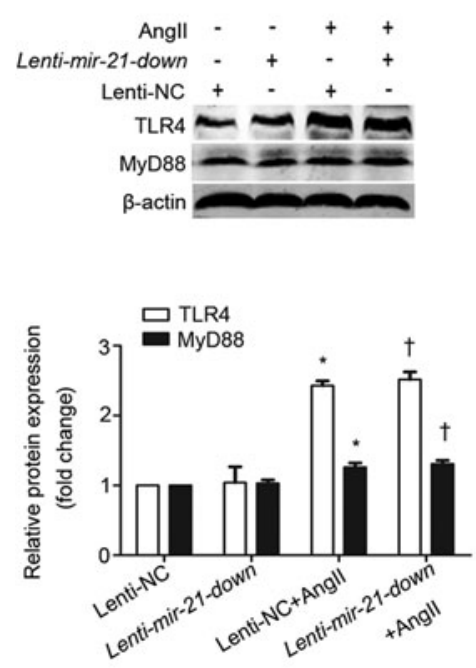

G

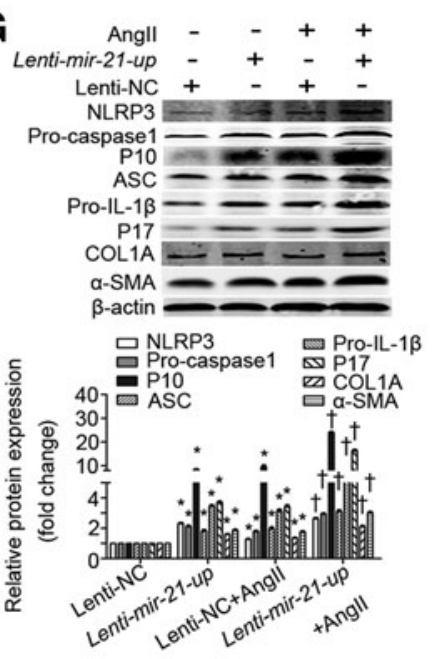

B

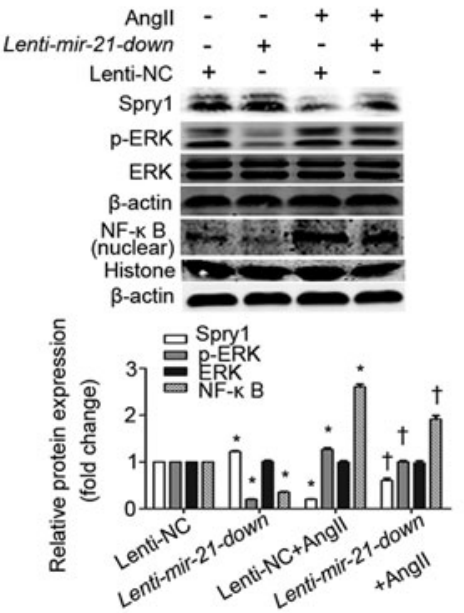

E
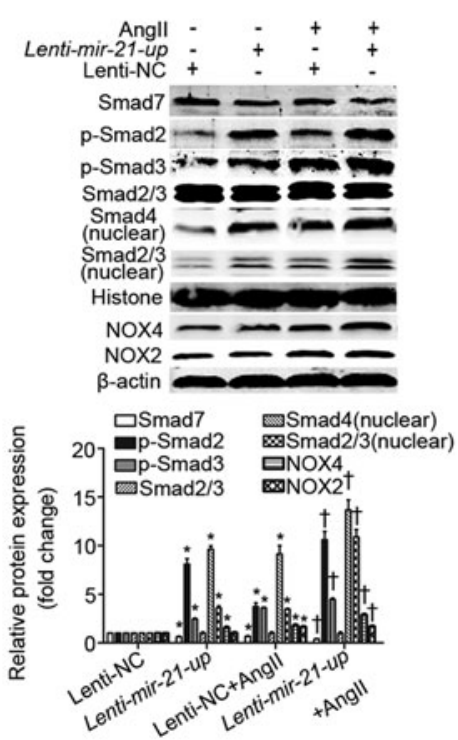

H
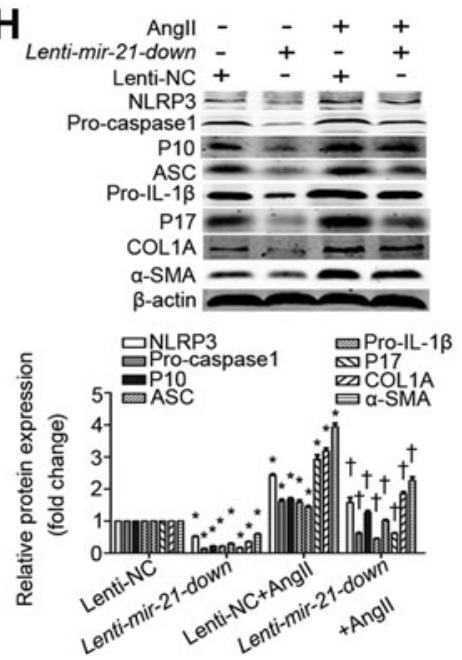

C
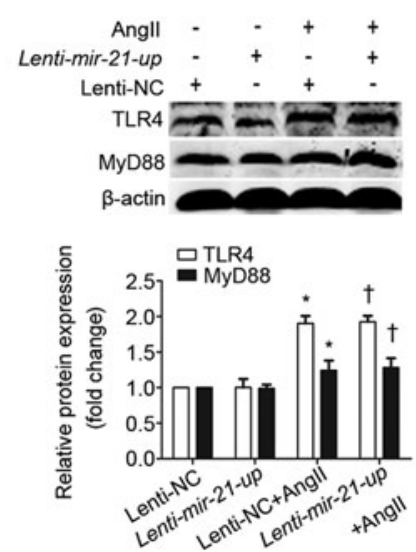

F

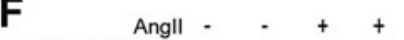
Lenti-mir-21-down - + ++ Lenti-NC + Smad7 $\rightleftharpoons$ p-Smad2 $\longrightarrow$

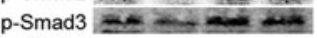
$\mathrm{Smad} 2 / 3=$ $\underset{\text { (nuclear) }}{\mathrm{Smad} 4}-\cdots$ $\underset{\text { (nuclear) }}{\mathrm{Smad2}} \mathrm{O}=$

Histone NOX4

$\mathrm{NOX} 2=-$

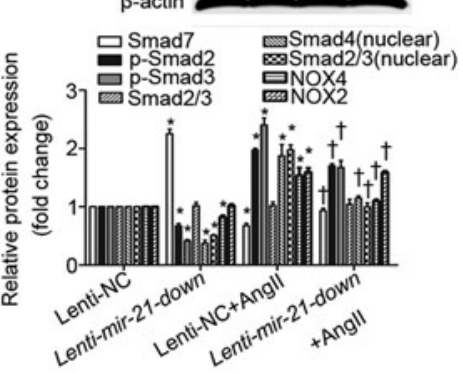

I
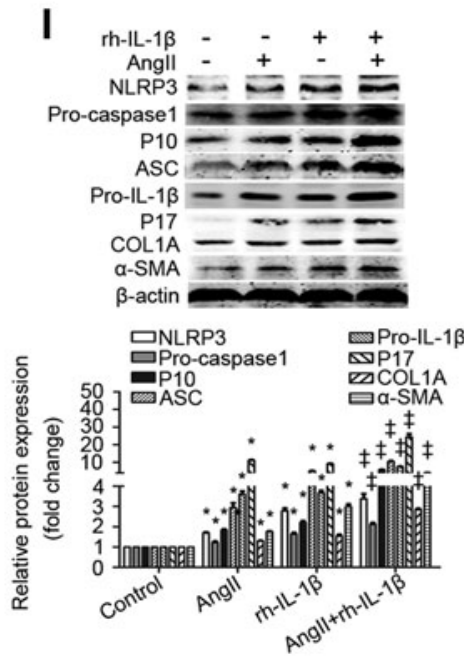

FIG. 10. Mir-21 mediated the effect of AngII on NLRP3 inflammasome activation via the Spry1/ERK/NF- $\kappa$ B and Smad7/Smad2/3/NOX4 pathways in HSCs. HSCs were transfected with lenti-mir-21-up or lenti-mir-21-down and treated with Ang II for $1 \mathrm{~h}$ (p-Smad2, p-Smad3, nucleoprotein NF- $\kappa$ B, Smad2/3, and Smad4) or for $24 \mathrm{~h}$. (A, B) Spry1, p-ERK, and NF- $\kappa$ B protein levels were analyzed by Western blot. (C, D) TLR4 and MyD88 protein levels were analyzed by Western blot. (E, F) Smad7, p-Smad2, p-Smad3, nucleoprotein Smad2/3, Smad4, NOX4, and NOX2 protein levels were analyzed by Western blot. (G, H) NLRP3 inflammasome, $\alpha$-SMA, and COL1A protein levels were analyzed by Western blot. The data are presented as mean \pm SEM. ${ }^{*} p<0.05$ versus lenti-NC; ${ }^{\dagger} p<0.05$ versus lenti-mir-21-up or lenti-mir-21-down. (I) HSCs were treated with rh-IL-1 $\beta(10 \mathrm{ng} / \mathrm{ml})$ and AngII $\left(10^{-7} M\right)$. NLRP3 inflammasome, $\alpha$-SMA, and COL1A protein levels were analyzed by Western blot. The data are presented as the mean \pm SEM. ${ }^{*} p<0.05$ versus control; ${ }^{\star} p<0.05$ versus AngII. All of the assays were performed in triplicate. TLR4, toll-like receptor 4. 
Mir-21 mediated the effect of Angll on NLRP3

inflammasome activation via the Spry1/ERK/NF-KB and Smad7/Smad2/3/NOX4 pathways in HSCs

First, we investigated the effects of mir-21 on the AngII-induced Spry1/ERK/NF- $\kappa$ B pathway. AngII treatment enhanced p-ERK protein expression and NF- $\kappa \mathrm{B}$ nucleoprotein levels but decreased Spry1 protein levels. Overexpression of mir-21 showed synergistic effects on AngII treatment. In contrast, inhibition of mir-21 expression reversed the effects of AngII (Fig. 10A, B).

As mentioned before, LPS primes signal 1 of the NLRP3 inflammasome; we investigated the effects of mir-21 on AngII-induced TLR4/MyD88/NF- $\kappa$ B pathway. The results showed that AngII increased TLR4 and MyD88 protein levels and promoted NF- $\kappa \mathrm{B}$ nuclear translocation. Overexpression or downregulation of mir-21 had no effect on AngII-induced increase in TLR4 and MyD88 protein levels but promoted or suppressed NF- $\kappa \mathrm{B}$ translocation (Fig. 10C, D and Supplementary Fig. S3A). These data indicate that mir-21 mediates AngII-induced activation of Spry1/ERK/NF- $\kappa$ B pathway rather than TLR4/MyD88/NF$\kappa \mathrm{B}$ pathway.

Next, we also found that AngII increased p-Smad2/3, NOX4, and NOX2 protein levels and promoted the Smad4 and Smad2/3 nuclear translocation but decreased Smad7 protein levels. Overexpression of mir-21 showed synergistic effects with the action of AngII on the molecules above, other than NOX2. In contrast, except NOX2, inhibition of mir-21 expression reversed the effects of AngII on the molecules mentioned above (Fig. 10E, F and Supplementary Fig. S3B). These data indicate that mir-21 mediates the AngII-induced activation of Smad7/Smad2/3/NOX4 pathway.

Furthermore, we found that AngII treatment increased NLRP3 inflammasome, COL1A, and $\alpha$-SMA protein levels. Overexpression of mir-21 showed synergistic effects with AngII treatment. In contrast, inhibition of mir-21 expression reversed the effects of AngII (Fig. 10G, H and Supplementary Fig. S4). These data indicate that mir-21 mediates AngIIinduced NLRP3 inflammasome.

Recombinant human IL- $1 \beta$ treatment showed synergistic effects with the AngII-induced increase in NLRP3 inflammasome, COL1A, and $\alpha$-SMA protein levels, suggesting that IL-1 $\beta$ increased collagen synthesis via NLRP3 inflammasome activation (Fig. 10I).

Taken together, these data demonstrate that mir-21 mediates the effect of AngII on NLRP3 inflammasome activation via the Spry1/ERK/NF- $\kappa \mathrm{B}$ and Smad7/Smad2/3/NOX4 pathways in HSCs (Fig. 11).

\section{Discussion}

In this article, we demonstrated for the first time that mir21 mediates AngII-induced activation of NLRP3 inflammasome and thereby collagen synthesis in HSCs via targeting Spryl and Smad7. Ang-(1-7) protected against BDL- and AngII-induced liver fibrosis and inhibited expression of mir21. The principal findings obtained include the following: (1) mir-21 is positively correlated with liver fibrosis and oxidation; (2) both ERK/NF- $\kappa$ B and Smad3/NOX/ROS pathways mediate AngII-induced overexpression of mir-21; (3) mir-21 activates the ERK/NF- $\kappa$ B and Smad2/3/NOX4/ROS pathways in HSCs via targeting Spry1, Smad7 for degradation,

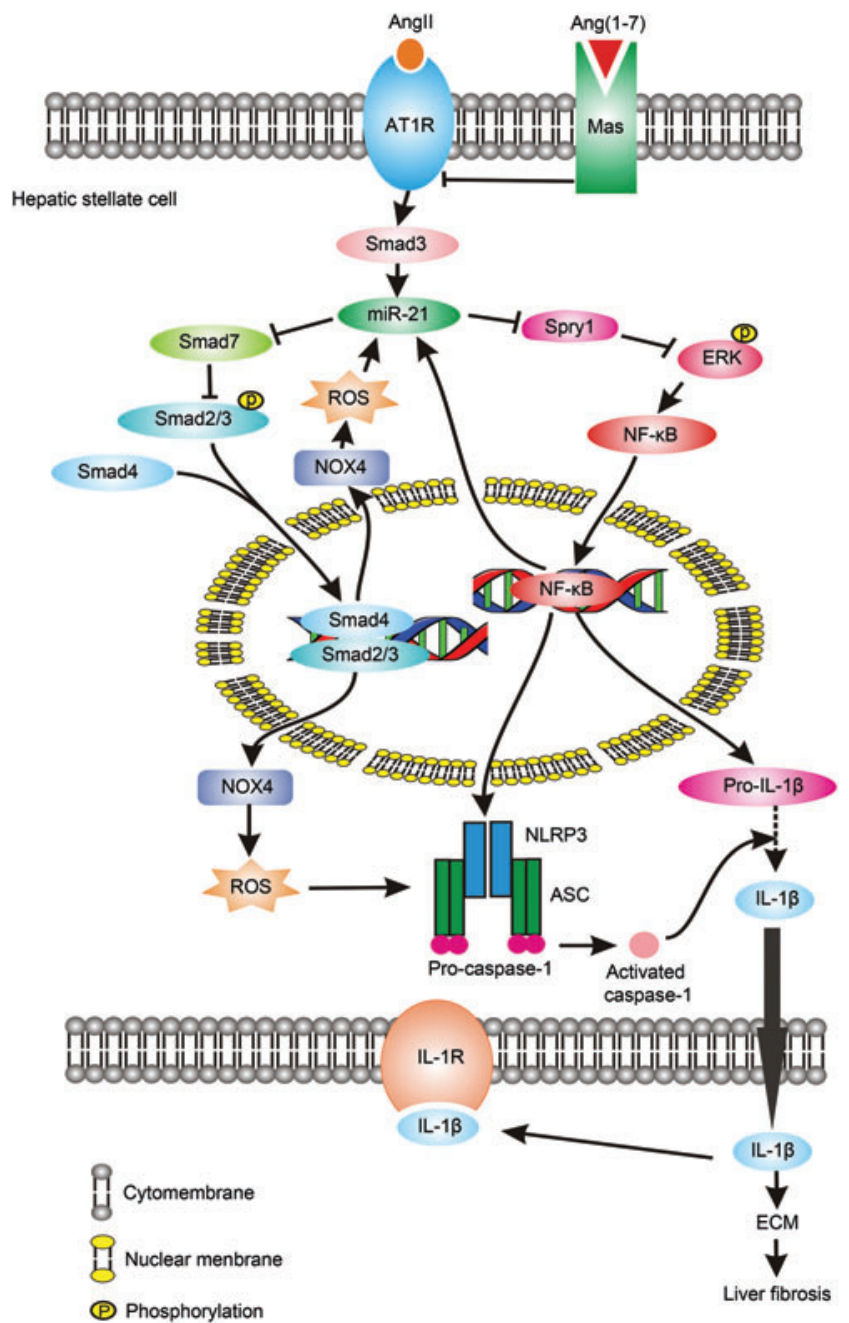

FIG. 11. A schematic view of major signal transduction pathways involved in the effects of mir-21 induced by Ang II on the NLRP3 inflammasome/IL-1 $\beta$ axis via activating the Spry1/ERK/NF- $\kappa$ B and Smad7/Smad2/3/ Smad4/NOX4 pathways. To see this illustration in color, the reader is referred to the web version of this article at www.liebertpub.com/ars

respectively; (4) AngII activated NLRP3 inflammasome/ IL-1 $\beta$ axis and thus promoted HSC activation via the mir-21/Spry 1/ERK/NF- $\kappa \mathrm{B}$ and mir-21/Smad7/Smad2/3/ NOX4 pathway, respectively; and (5) Ang-(1-7) protected against BDL- or AngII-induced liver fibrosis and inhibited mir-21 expression.

A recent study has shown that the mir-21 level is upregulated in fibrotic liver biopsies of HCV patients (24) and mir-21 overexpression mediates the activation of HSCs (12, 48). Consistent with these reports, our in vitro data showed that overexpression of mir-21 promoted oxidation, mitochondrial dysfunction, and HSC activation. However, downregulation of mir-21 exerted the opposite effects. In vivo data showed a markedly increased mir-21 level in blood and liver tissues of patients with liver cirrhosis or rats with BDL- or AngII-induced hepatic fibrosis. Meanwhile, lenti-mir-21-down attenuated liver fibrosis and the oxidation induced by AngII. It is noteworthy that there is a positive correlation of human circulating mir-21 levels with liver 
FibroScan value, suggesting that circulating mir-21 could potentially serve as a biomarker in patients with liver fibrosis. Hence, mir-21 is a promising biomarker and a therapeutic target for liver fibrosis.

Some studies have reported that AngII regulated cardiac fibrosis via mir-21 induction. The work by Siddesha et al. (32) found that AngII promotes cardiac fibroblast migration and thereby cardiac fibrosis by suppressing RECK expression, a critical molecular for cardiac fibroblast migration, via ERK-dependent AP-1- and STAT3-mediated mir-21 induction. Moreover, AngII suppressed the proapoptotic PTEN and Spry1, but induced the MMP2. Also, the work by Adam et al. (2), Maegdefessel et al. (23), and Lorenzen et al. (21) provided similar insights. However, a recent report showed that AngII upregulated the expression of mir-224 but not mir21 in adult rat cardiac fibroblasts (27). Considering these findings in cardiac fibrosis, we investigated whether AngII could promote liver fibrosis by regulating mir-21 and the detail mechanism. We found that AngII treatment induced an increase in mir-21 in HSCs and liver tissue. Meanwhile, AngII treatment elevated mir-21 expression in HSCs in a time dependency but not dose dependency. AngII $10^{-7} M$ had the best effect to promote mir-21 expression. The increase in mir-21 induced by AngII in HSCs could be reduced by siSmad3 (not by si-Smad2) and antioxidants VAS2870, NAC, catalase, and mito-TEMPO. Moreover, blockade of ERK and $\mathrm{NF}-\kappa \mathrm{B}$ prevented AngII-induced mir-21 expression. This result is consistent with Ling's report that activation of ERK/ NF- $\kappa$ B promoted mir-21 expression (19). Hence, Smad3/ NOX/ROS and ERK/NF- $\kappa \mathrm{B}$ pathways mediate AngIIinduced mir-21 expression in HSCs.

Furthermore, it remains to be determined how mir-21 simultaneously regulates multiple signaling pathways in hepatic fibrogenesis. In the present study, we focused on two target genes of mir-21, namely Spryl and Smad7.

Previous studies have shown that mir-21 contributes to fibrosis along with degradation of Spryl to stimulate ERK signaling in cardiac fibroblasts (38) and HSCs (49). In the present study, mir-21 overexpression promoted Spryl degradation, enhanced p-ERK levels, and induced NF- $\kappa \mathrm{B}$ nuclear translocation in HSCs. In addition, in vivo data also demonstrated a markedly increased p-ERK and nucleoprotein NF- $\kappa$ B but decreased Spry 1 protein expression in hepatic tissues of patients with liver cirrhosis and rats with hepatic fibrosis induced by BDL or AngII. Hence, by targeting Spryl for degradation, AngII-induced mir-21 mediated the ERK/ $\mathrm{NF}-\kappa \mathrm{B}$ pathway in HSCs.

We then focused on the Smad2/3/NOX4/ROS pathway. Being a "constitutively active" enzyme, NOX4 contributes to generation of $\mathrm{H}_{2} \mathrm{O}_{2}$. NOX4-derived ROS are a critical factor in HSC activation and hepatic fibrogenesis (30). TGF$\beta$ induces the Smad2/3 complex with Smad 4 and translocates to the nucleus, leading to NOX4 gene transcription and NOX4-derived ROS generation (35). Our recent report (4) further showed that Smad3 gene depletion inhibits NOX4 protein levels and that NOX4 gene depletion reduces Smad3 protein levels, suggesting the feedback loop between NOX4 and Smad3. Hence, we deduced the mechanism of mir-21 inducing HSC activation: via targeting Smad7 for degradation, mir-21 promotes the Smad2/3/NOX4/ROS pathway.

Our findings confirm the suggestion. We found that AngIIinduced mir-21 overexpression degraded Smad7 and subse- quently promoted $\mathrm{Smad} 2 / 3$ phosphorylation and the recruitment of Smad2/3 with Smad4 in the nucleus, thus leading to increase of NOX4 protein level and $\mathrm{H}_{2} \mathrm{O}_{2}$ generation, which could be blocked by Smad3 inhibitor SB43152.

Noteworthy, NOX2 is also an important isoform of NOX family. NOX2 was implicated in the pathology of AngII (5, $13,18,26)$ and liver fibrogenesis $(14,29)$. In consistence with these, here we found that AngII increased both NOX2 and NOX4 protein levels in HSCs, and NOX inhibitor VAS2870, which shows the highest affinity for NOX2 (3), inhibited AngII-induced mir-21 overexpression and resultant inhibition of $\mathrm{H}_{2} \mathrm{O}_{2}$ generation. Hence, NOX2 actually involves in the process above. However, mir- 21 overexpression resulted in increased protein expression level of NOX4, other than NOX2. Overexpression of mir-21 enhanced NOX4 protein expression induced by AngII. Inhibition of mir-21 expression reduced NOX4 protein expression level induced by AngII. In contrast, mir- 21 could not regulate NOX2 protein level induced by AngII, indicating that not NOX2 but NOX4 mediates oxidative stress initiated by mir-21 overexpression. The reason may be associated with the feedback loop between NOX4 and Smad3 (4). Whether NOX2 can be regulated by $\mathrm{Smad} 2 / 3$ remains unclear, which deserves further investigation.

Moreover, mir-21 overexpression induced mitochondrial dysfunction and promoted mito-ROS generation in HSCs. In contrast, downregulation of mir-21 exerted the opposite effects. Interestingly, mir-21 promoted NOX4 translocation into the mitochondria, indicating cross talk between the mitochondria and NOX4 in HSCs. In agreement with the results in vitro, in vivo data indicated a markedly high level of mir-21, along with increased Smad2/3, NOX4 protein levels, and $\mathrm{H}_{2} \mathrm{O}_{2}$ production in the fibrotic liver tissues of humans or rats treated with BDL or AngII infusion. Meanwhile, lenti-mir-21-down attenuated the effects induced by AngII infusion. Therefore, by targeting Smad7 for degradation, AngII-induced mir-21 promoted generation of ROS (including NOX-derived ROS and mito-ROS) in the HSCs via the Smad2/3-NOX pathway.

Interestingly, ERK promoted mir-21 expression, and mir21 caused feedback activation of ERK by targeting Spry1, whereas the Smad3/NOX/ROS pathway mediated AngIIinduced mir-21 expression and mir-21 caused feedback activation of Smad3/NOX/ROS by targeting Smad7. We demonstrated that mir-21 maintains itself at constant high levels and drives forces for hepatic fibrosis using two feedback loops: mir-21/Spry1/ERK loop and mir-21/Smad7/ Smad3/NOX/ROS loop. Targeting these aberrantly activated feedback loops may provide new therapeutic strategies against hepatic fibrosis.

The NLRP3 inflammasome is a novel mediator for liver fibrosis (36). We found that injection of lenti-mir-21-down attenuated AngII-induced activation of the NLRP3 inflammasome/IL-1 $\beta$ axis in the rat liver. Nevertheless, how mir-21 regulates NLRP3 inflammasome activation has not been reported until now. This study is the first that connects two target genes of mir-21 with two signals of the NLRP3 inflammasome.

Cell priming with an NF- $\kappa \mathrm{B}$ activator, such as TLR4ligand LPS, is the signal 1 of NLRP3 inflammasome activation. The TLR/MyD88/NF- $\kappa$ B pathway mediates NLRP3 and proIL-1 $\beta$ gene transcription, whereas signal 2 , triggered by a broad 
variety of DAMP molecules such as ROS, induces the assembly of NLRP3 inflammasome complex, resulting in activation of caspase- 1 and subsequent pro-IL-1 $\beta$ maturation (36).

In this study, AngII-induced mir-21 promoted ERK phosphorylation and nuclear translocation of NF- $\kappa \mathrm{B}$ via inhibition of the target gene Spry1 but did not affect TLR4 and Myd88. U0126 or BAY, inhibitor of ERK or NF- $\kappa$ B, inhibited activation of NLRP3 inflammasome complex induced by mir- 21 overexpression. Hence, our study is the first to demonstrate that the signal 1 of NLRP3 inflammasome was activated by AngII via the mir-21/Spry1/ERK/NF- $\kappa$ B pathway. The mir-21/Spry $1 /$ ERK/NF- $\kappa \mathrm{B}$ pathway is a new manner of regulation of NLRP3 inflammasome.

As for signal 2, we demonstrate, for the first time, that ROS generation regulated by the mir-21/Smad7/Smad3/NOX4 pathway mediates AngII-induced activation of NLRP3 inflammasome. We found that overexpression of mir-21 increased protein levels and the assembly of the NLRP3 inflammasome complex, as well as a marked increase in Smad2/3 phosphorylation, $\mathrm{H}_{2} \mathrm{O}_{2}$ content, and colocalization of NLRP3 and NOX4 in the mitochondria. Smad3 inhibitor SB43152, VAS2870, and NAC reduced the increase in NLRP3 inflammasome complex protein levels induced by mir-21 overexpression. Overexpression of mir-21 had synergistic effects with AngII-induced increase in NLRP3 inflammasome. In contrast, inhibition of mir-21 expression reversed the effects of AngII. These data indicate that the mir-21/Smad7/Smad2/3/NOX4 pathway mediates AngIIinduced NLRP3 inflammasome activation.

Moreover, mito-TEMPO also inhibited NLRP3 inflammasome complex protein levels induced by mir- 21 overexpression. Hence, besides NOX4-derived ROS, mito-ROS mediated mir-21-induced NLRP3 inflammasome activation.

Then, we come to the final result of NLRP3 inflammasome activation. In our study, overexpression of mir-21 induced a marked increase in P17 (an active part of IL-1 $\beta$ ), COL1A, and $\alpha$-SMA. NLRP3 depletion inhibited expression of P17, COL1A, and $\alpha$-SMA induced by mir- 21 . Moreover, recombinant rat IL- $1 \beta$ exerted a synergistic effect with mir- 21 on COL1A and $\alpha$-SMA production. Injection with lenti-mir-21down attenuated liver fibrosis and AngII-induced activation of NLRP3 inflammasome/IL- $1 \beta$ axis in the rat liver. Because mir-21 triggers two signals of NLRP3 inflammasome/IL- $1 \beta$ axis, downregulating mir-21 is an effective strategy for inhibition of hepatic fibrosis.

Taken together, we demonstrate that AngII activates NLRP3 inflammasome/IL- $1 \beta$ axis and thus promotes HSC activation via the mir-21/Spry1/ERK/NF- $\kappa \mathrm{B}$, mir-21/Smad7/ Smad2/3/NOX4 pathway.

Finally, we come to the effects of Ang-(1-7) on mir-21. Ang(1-7) has been shown to attenuate liver fibrosis in BDL rats (31). Recently, we reported that Ang-(1-7) inhibits HSC activation by inhibiting NLRP3 inflammasome activation in HSCs (4). Acuna et al. showed that Ang-(1-7) reduced expression of mir-21 in skeletal muscle fibroblasts (1). However, the precise mechanisms underlying Ang(1-7) attenuating liver fibrosis by inhibiting mir-21 remain unclear. In the present study, Ang-(1-7) inhibited either mir-21 expression or AngII-induced mir-21 expression in vitro. In vivo, exogenous Ang-(1-7) infusion significantly decreased $\mathrm{mir}-21$ expression, NLRP3 inflammasome activation, and attenuated liver fibrosis induced by BDL or AngII infusion. Hence, we first reported that Ang-(1-7) decreases mir-21 expression in vitro and in vivo, suggesting the potential mechanism of Ang-(1-7) treatment against liver fibrosis. A more detailed mechanism deserves further investigation.

So far, there are some clinical data to support the use of the RAS inhibitor, such as angiotensin II type 1 receptor blocker or angiotensin converting enzyme inhibitor in human liver fibrosis $(7,33,37,44,45)$. These studies have administered the drug alone, or in combination with others. These clinical studies potently support the clinical impact of the here reported mechanism for the clinical situation.

In conclusion, we identified AngII-induced mir-21 as a crucial factor that initiates HSC activation by upregulating NLRP3 inflammasome/IL- $\beta$ axis via the Spry1/ERK/NF$\kappa \mathrm{B}$ and $\mathrm{Smad} 7 / \mathrm{Smad} 2 / 3 / \mathrm{NOX} 4$ pathways. Ang-(1-7) protected against BDL- and AngII-induced liver fibrosis and inhibited expression of mir-21. Consequently, the present study revealed a specific mechanism underlying AngII-induced mir-21 activating HSCs, suggesting that downregulation of mir-21 is a promising strategy for treatment of hepatic fibrosis.

\section{Materials and Methods}

\section{Reagents}

Ang-(1-7), AngII, A779(Mas blocker), SB431542 (Smad3 blocker), VAS2870 (NADPH-oxidase blocker), N-acetylL-cysteine (NAC, a superoxide inhibitor), mito-TEMPO, polyethylene glycol-adsorbed-superoxide dismutase (PEGSOD), and catalase were provided by Sigma-Aldrich (SigmaAldrich, St. Louis, MO). rh-IL-1 $\beta$ was obtained through ProSpec Protein Specialists (Rehovot, Israel). ALZET osmotic pumps (models 2004 and 2ML4) were provided by the DURECT Corporation (Cupertino, CA). All of the siRNA was synthesized by GenePharma (Shanghai, China). All of the primers were provided by Invitrogen (Shanghai, China). Lenti NC, mir-21 virus, Smad7, and Spryl plasmids were obtained through GeneChem (Shanghai, China).

\section{Human blood and liver specimens}

Control liver tissues were obtained from 12 patients undergoing partial liver resection for hepatic hemangioma. Normal blood samples were obtained from 12 normal persons. Fibrotic liver biopsy specimens and abnormal blood samples were obtained from 12 patients with liver fibrosis (8 patients suffered from HBV infection, 4 patients suffered from bile duct stones) who underwent a liver hardness scan (FibroScan value $>7.3 \mathrm{kPa}$ ) by FibroScan (ECHOSENS Company, France). Each person had signed the informed written consent, and the Ethics Committee at the Nanfang hospital had approved the use of the samples.

\section{Animal model}

Male Wistar rats (200-300 g) were obtained from the Laboratory Animal Center (Southern Medical University, China). The Committee on the Ethics of Animal Experiments of Southern Medical University had approved all experimental procedures on rats (Permission No. SCXK 2009-015). The rats were housed under an environment (12-h light/12-h dark; $22-24^{\circ} \mathrm{C}$ ) and received water ad libitum. In the first animal model, the model of hepatic fibrosis was established by BDL. Mini-Osmotic pumps containing saline or Ang-(1-7) 
$\left(25 \mu \mathrm{g} \cdot \mathrm{kg}^{-1} \cdot \mathrm{h}^{-1}\right)$ were implanted into the abdominal cavity of rats. There were three groups (9 rats/group): the sham group, the BDL+saline group, and the BDL+Ang-(1-7) group. In the second animal model, liver fibrosis was induced by continuous infusion of AngII $\left(25 \mu \mathrm{g} \cdot \mathrm{kg}^{-1} \cdot \mathrm{h}^{-1}\right)$ with miniOsmotic pumps, and control rats received an infusion of saline. Mir-21 downexpression lentivirus was injected into the tail vein. Animals were divided into the sham group, the AngII group, the AngII+lenti-mir-2l-NC group, and the AngII+lenti-mir-21-down group, each group containing nine rats. In the third model of liver fibrosis group, there were four groups, each containing nine rats: the control group, the AngII group, the Ang-(1-7) group, and the AngII+Ang-(1-7) group. All rats were sacrificed 4 weeks later.

\section{Cell culture}

Rat HSCs were isolated and cultured in Dulbecco's modified Eagle's medium (GIBCO BRL, Life Technologies, Inc.). HEK-293 cells were obtained from the cell bank of the Chinese Academy of Sciences in Shanghai. Experiments were performed on cells at passages 3-5.

\section{Histological analysis}

For IHC analysis, the sections were incubated with antibody against $\alpha 1$-type 1 collagen (COL1A), $\alpha$-SMA, VWF, NOX4, NLRP3, caspase-1, IL-1 $\beta$ (1:100; Abcam, Cambridge, MA), Smad7, Spry1 (1:50; Santa Cruz Biotechnology).

\section{In situ hybridization}

In brief, the livers sections were treated with acetylation solution and proteinase $\mathrm{K}$. Then, the sections were blocked with hybridization solution and incubated with digoxigeninconjugated mir-21 probes (Exiqon, Denmark). Then, the sections were incubated with the HRP-conjugated antidigoxigenin antibody (Roche, Shanghai, China). Finally, the sections were treated with NBT/BCIP (Roche, Shanghai, China). Light blue cytoplasmic staining means positive.

\section{$R N A$ isolation and $q-R T-P C R$}

Total RNA was extracted by using TRIzol. Blood total RNA was isolated from $200 \mu$ l of blood using the miRNeasy Mini Kit (TransGene Biotech, Beijing, China). One thousand nanograms of tissue or cell RNA was reverse transcribed and performed q-PCR using the All-in-One mir-21 q-RT-PCR kit (GeneCopoeia, Guangzhou, China). Blood RNA $(2.5 \mu \mathrm{l})$ was reverse transcribed and performed q-PCR using the miRNeasy Mini Kit. Real-time PCR was performed using the ABI 7500 Real-Time PCR System (Applied Biosystems). Mir-21 primer: UAGCUUAUCAGACUGAUGUUGA, the universal primer was provided by All-in-One miR-21 q-RTPCR kit or miRNeasy Mini Kit. Mir-21 expression was normalized using the $2^{-\Delta \Delta \mathrm{Ct}}$ method from the $\mathrm{Ct}$ values of the respective miRNAs relative to the housekeeping gene $U 6$ snRNA.

\section{Western blotting}

The proteins level was detected by Western blotting. The primary antibodies were $\alpha$-SMA, NOX4, NOX2, NLRP3, caspase1, ASC, IL-1 $\beta$ (1:1000; Abcam, Cambridge, MA),
p-Smad2, p-Smad3, Smad2/3 (1:1000; Cell Signaling Technology, MA), COL1A, Smad7, Spry1 (1:100; Santa Cruz Biotechnology). $\beta$-Actin acted as an internal control.

\section{Transfection studies}

Mir-21 NC and overexpression or downexpression of lentivirus were transfected according to the manufacturer's instructions. The siRNA sequences: $\operatorname{Smad} 3$ (sense: $5^{\prime}-\mathrm{CCG}$ CAUGAGCUUCGUCAAATT-3', antisense: $5^{\prime}$-UUUGACG AAGCUCAUGCGGTT-3'); Smad2 (sense: $5^{\prime}$-AATAATAT AAGCGCACTCCTCCCTGTCTC-3', antisense: 5'-AAGA GGAGTGCGCTTATATTACCTGTCTC-3'); and NLRP3 (sense: $5^{\prime}$-GCUUCAGCCACAUGACUUU-3', antisense: 5' AAAGUCAUGUGGCUGAAGC-3').

\section{Luciferase reporter assay}

Cells were cotransfected with $0.5 \mu \mathrm{g}$ wild-type or mutanttype $3^{\prime}$-UTR luciferase reporters containing the firefly luciferase gene and Renilla luciferase gene and lenti-mir-21-NC or lenti-mir-21-up into HEK-293T cells. Renilla luciferase reporters were also included as an internal control.

\section{Hepatic hydroxyproline determination}

The hydroxyproline detection kit (Jiancheng Institute of Biotechnology, Nanjing, China) was used to measure the hepatic hydroxyproline content.

\section{Hydrogen peroxide assay}

The $\mathrm{H}_{2} \mathrm{O}_{2}$ product was detected using the Hydrogen Peroxide Assay Kit (Beyotime, Shanghai, China). The technical principle of the used Hydrogen Peroxide Assay Kit is that a ferrous iron can be oxidized to ferric iron by $\mathrm{H}_{2} \mathrm{O}_{2}$ and then react with xylenol orange in a specific solution to synthesizing purse products. So, the concentration of the $\mathrm{H}_{2} \mathrm{O}_{2}$ can be detected by microplate reader. The cells were cracked in lysis buffer, and then, hydrogen peroxide detection of liquid was added, followed by supernatant and standard substance. Finally, the OD value was detected at $562 \mathrm{~nm}$ wavelength absorption spectroscopy.

\section{Intracellular superoxide and mitochondrial ROS measurement}

The fluorescent probe dihydroethidium (Beyotime, Shanghai, China) was used. Mito-ROS was detected by measuring mitochondrial superoxide with the fluorogenic probe MitoSOX red reagent (M36008; Invitrogen Life Technologies). PEG-SOD was used to determine the specificity of MitoSOX red oxidation for measurements of superoxide.

\section{Intracellular ATP level assay}

The intracellular ATP levels were measured according to instructions (Beyotime, Shanghai, China).

\section{Statistical analysis}

All experiments were repeated at least three times. Data are expressed as the mean $\pm \mathrm{SD}$. Data were analyzed by ANOVA with LSD for multiple comparisons. For association studies, Pearson analysis was used for parametric and 
Spearman was used for nonparametric data. $r>0$ was considered a positive correlation. Differences were considered significant at a $p<0.05$. The data were analyzed using SPSS 13.0.

\section{Acknowledgments}

We thank Prof. Pingsheng Wu, Prof. Zhenshu Zhang, and Prof. Xishan Yang for their kind help in this study. The study was supported by the National Natural Science Foundation of China (81070338), Guangdong Province Natural Science Foundation of China (S2012010009322), the Team Program of Natural Science Foundation of Guangdong Province, China (S2011030003134), the specialized research fund for the doctoral program of higher education jointly funded projects (20124433110004), Guangzhou Pilot Project of Clinical and Translational Research Center (early gastrointestinal cancers, No. 7415696196402), and Guangdong Provincial Bio-engineering Research Center for Gastroenterology Disease.

\section{Author Disclosure Statement}

No competing financial interests exist.

\section{References}

1. Acuna MJ, Pessina P, Olguin H, Cabrera D, Vio CP, Bader M, Munoz-Canoves P, Santos RA, Cabello-Verrugio C, and Brandan E. Restoration of muscle strength in dystrophic muscle by angiotensin-1-7 through inhibition of TGF-beta signalling. Hum Mol Genet 23: 1237-1249, 2014.

2. Adam O, Löhfelm B, Thum T, Gupta SK, Puhl SL, Schäfers HJ, Böhm M, and Laufs U. Role of miR-21 in the pathogenesis of atrial fibrosis. Basic Res Cardiol 107: 278, 2012.

3. Altenhöfer S, Kleikers PW, Radermacher KA, Scheurer P, Rob Hermans JJ, Schiffers P, Ho H, Wingler K, and Schmidt HH. The NOX toolbox: validating the role of NADPH oxidases in physiology and disease. Cell Mol Life Sci 69: 2327-2343, 2012.

4. Cai SM, Yang RQ, Li Y, Ning ZW, Zhang LL, Zhou GS, Luo W, Li DH, Chen Y, Pan MX, and Li X. Angiotensin(1-7) improve liver fibrosis by regulating the NLRP3 inflammasome via redox balance modulation. Antioxid Redox Signal 24: 795-812, 2016.

5. Chrissobolis S, Banfi B, Sobey CG, and Faraci FM. Role of Nox isoforms in angiotensin II-induced oxidative stress and endothelial dysfunction in brain. J Appl Physiol 113: 184191, 2012.

6. Daiber A. Redox signaling (cross-talk) from and to mitochondria involves mitochondrial pores and reactive oxygen species. Biochim Biophys Acta 1797: 897-906, 2010.

7. Debernardi-Venon W, Martini S, Biasi F, Vizio B, Termine A, Poli G, Brunello F, Alessandria C, Bonardi R, Saracco G, Rizzetto M, and Marzano A. AT1 receptor antagonist Candesartan in selected cirrhotic patients: effect on portal pressure and liver fibrosis markers. J Hepatol 46: 10261033, 2007.

8. Dikalova AE, Bikineyeva AT, Budzyn K, Nazarewicz RR, McCann L, Lewis W, Harrison DG, and Dikalov SI. Therapeutic targeting of mitochondrial superoxide in hypertension. Circ Res 107: 106-116, 2010.
9. Fraga-Silva RA, Da Silva DG, Montecucco F, Mach F, Stergiopulos N, da Silva RF, and Santos RA. The angiotensin-converting enzyme 2/angiotensin-(1-7)/Mas receptor axis: a potential target for treating thrombotic diseases. Thromb Haemost 108: 1089-1096, 2012.

10. Friedman SL. Mechanisms of hepatic fibrogenesis. Gastroenterology 134: 1655-1669, 2008.

11. Guzik TJ, Hoch NE, Brown KA, McCann LA, Rahman A, Dikalov S, Goronzy J, Weyand C, and Harrison DG. Role of the $\mathrm{T}$ cell in the genesis of angiotensin II induced hypertension and vascular dysfunction. J Exp Med 204: 24492460, 2007.

12. He X, Xie J, Zhang D, Su Q, Sai X, Bai R, Chen C, Luo X, Gao G, and Pan W. Recombinant adeno-associated virusmediated inhibition of microRNA-21 protects mice against the lethal schistosome infection by repressing both IL-13 and transforming growth factor beta 1 pathways. Hepatology 61: 2008-2017, 2015.

13. Bendall JK, Rinze R, Adlam D, Tatham AL, de Bono J, and Channon KM. Endothelial Nox 2 overexpression potentiates vascular oxidative stress and hemodynamic response to angiotensin II: studies in endothelial-targeted Nox2 transgenic mice. Circ Res 100: 1016-1025, 2007.

14. Jiang JX, Venugopal S, Serizawa N, Chen X, Scott F, Li Y, Adamson R, Devaraj S, Shah V, Gershwin ME, Friedman SL, and Török NJ. Reduced nicotinamide adenine dinucleotide phosphate oxidase 2 plays a key role in stellate cell activation and liver fibrogenesis in vivo. Gastroenterology 139: 1375-1384, 2010.

15. Kimura S, Zhang GX, Nishiyama A, Shokoji T, Yao L, Fan YY, Rahman M, and Abe Y. Mitochondria-derived reactive oxygen species and vascular MAP kinases: comparison of angiotensin II and diazoxide. Hypertension 45: 438-444, 2005.

16. Kossmann S, Schwenk M, Hausding M, Karbach SH, Schmidgen MI, Brandt M, Knorr M, Hu H, Kröller-Schön S, Schönfelder T, Grabbe S, Oelze M, Daiber A, Münzel T, Becker C, and Wenzel P. Angiotensin II-induced vascular dysfunction depends on interferon- $\gamma$-driven immune cell recruitment and mutual activation of monocytes and NKcells. Arterioscler Thromb Vasc Biol 33: 1313-1319, 2013.

17. Kröller-Schön S, Steven S, Kossmann S, Scholz A, Daub S, Oelze M, Xia N, Hausding M, Mikhed Y, Zinssius E, Mader M, Stamm P, Treiber N, Scharffetter-Kochanek K, Li H, Schulz E, Wenzel P, Münzel T, and Daiber A. Molecular mechanisms of the crosstalk between mitochondria and NADPH oxidase through reactive oxygen speciesstudies in white blood cells and in animal models. Antioxid Redox Signal 20: 247-266, 2014.

18. Landmesser U, Cai H, Dikalov S, McCann L, Hwang J, Jo $\mathrm{H}$, Holland SM, and Harrison DG. Role of $\mathrm{p} 47^{\mathrm{phox}}$ in vascular oxidative stress and hypertension caused by angiotensin II. Hypertension 40: 511-515, 2002.

19. Ling M, Li Y, Xu Y, Pang Y, Shen L, Jiang R, Zhao Y, Yang X, Zhang J, Zhou J, Wang X, and Liu Q. Regulation of miRNA-21 by reactive oxygen species-activated ERK/ NF-kappaB in arsenite-induced cell transformation. Free Radic Biol Med 52: 1508-1518, 2012.

20. Liu G, Friggeri A, Yang Y, Milosevic J, Ding Q, Thannickal VJ, Kaminski N, and Abraham E. miR-21 mediates fibrogenic activation of pulmonary fibroblasts and lung fibrosis. J Exp Med 207: 1589-1597, 2010.

21. Lorenzen JM, Schauerte C, Hubner A, Kolling M, Martino F, Scherf K, Batkai S, Zimmer K, Foinquinos A, Kaucsar T, Fiedler J, Kumarswamy R, Bang C, Hartmann D, Gupta 
SK, Kielstein J, Jungmann A, Katus HA, Weidemann F, Muller OJ, Haller H, and Thum T. Osteopontin is indispensible for AP1-mediated angiotensin II-related miR-21 transcription during cardiac fibrosis. Eur Heart J 36: 2184 2196, 2015.

22. Lubel JS, Herath CB, Tchongue J, Grace J, Jia Z, Spencer K, Casley D, Crowley P, Sievert W, Burrell LM, and Angus PW. Angiotensin-(1-7), an alternative metabolite of the renin-angiotensin system, is up-regulated in human liver disease and has antifibrotic activity in the bile-duct-ligated rat. Clin Sci (Lond) 117: 375-386, 2009.

23. Maegdefessel L, Azuma J, Toh R, Deng A, Merk DR, Raiesdana A, Leeper NJ, Raaz U, Schoelmerich AM, McConnell MV, Dalman RL, Spin JM, and Tsao PS. MicroRNA-21 blocks abdominal aortic aneurysm development and nicotine-augmented expansion. Sci Transl Med 4: 122ra22, 2012.

24. Marquez RT, Bandyopadhyay S, Wendlandt EB, Keck K, Hoffer BA, Icardi MS, Christensen RN, Schmidt WN, and McCaffrey AP. Correlation between microRNA expression levels and clinical parameters associated with chronic hepatitis C viral infection in humans. Lab Invest 90: 1727-1736, 2010.

25. Meng Y, Li T, Zhou GS, Chen Y, Yu CH, Pang MX, Li W, Li Y, Zhang WY, and Li X. The angiotensin-converting enzyme 2/angiotensin (1-7)/Mas axis protects against lung fibroblast migration and lung fibrosis by inhibiting the NOX4-derived ROS-mediated RhoA/Rho kinase pathway. Antioxid Redox Signal 22: 241-258, 2015.

26. Murdoch CE, Alom-Ruiz SP, Wang M, Zhang M, Walker $\mathrm{S}, \mathrm{Yu} \mathrm{B}$, Brewer A, and Shah AM. Role of endothelial Nox2 NADPH oxidase in angiotensin II-induced hypertension and vasomotor dysfunction. Basic Res Cardiol 106: 527-538, 2011.

27. Ning Q, and Jiang X. Angiotensin II upregulated the expression of microRNA-224 but not microRNA-21 in adult rat cardiac fibroblasts. Biomed Rep 1: 776-780, 2013.

28. Oakley F, Teoh V, Ching ASG, Bataller R, Colmenero J, Jonsson JR, Eliopoulos AG, Watson MR, Manas D, and Mann DA. Angiotensin II activates I kappaB kinase phosphorylation of RelA at Ser 536 to promote myofibroblast survival and liver fibrosis. Gastroenterology 136: 2334 2344.e1, 2009.

29. Paik YH, Iwaisako K, Seki E, Inokuchi S, Schnabl B, Osterreicher $\mathrm{CH}$, Kisseleva T, and Brenner DA. The nicotinamide adenine dinucleotide phosphate oxidase (NOX) homologues NOX1 and NOX2/gp91(phox) mediate hepatic fibrosis in mice. Hepatology 53: 1730-1741, 2011.

30. Paik YH, Kim J, Aoyama T, De Minicis S, Bataller R, and Brenner DA. Role of NADPH oxidases in liver fibrosis. Antioxid Redox Signal 20: 2854-2872, 2014.

31. Pereira RM, Dos Santos RA, Teixeira MM, Leite VH, Costa LP, da Costa Dias FL, Barcelos LS, Collares GB, and Simoes e Silva AC. The renin-angiotensin system in a rat model of hepatic fibrosis: evidence for a protective role of Angiotensin-(1-7). J Hepatol 46: 674-681, 2007.

32. Siddesha JM, Valente AJ, Yoshida T, Sakamuri SS, Delafontaine $\mathrm{P}$, Iba $\mathrm{H}$, Noda $\mathrm{M}$, and Chandrasekar B. Docosahexaenoic acid reverses angiotensin II-induced RECK suppression and cardiac fibroblast migration. Cell Signal 26: 933-941, 2014.

33. Sookoian S, Fernández MA, and Castaño G. Effects of six months losartan administration on liver fibrosis in chronic hepatitis C patients: a pilot study. World J Gastroenterol 11: 7560-7563, 2005.
34. Strowig T, Henao-Mejia J, Elinav E, and Flavell R. Inflammasomes in health and disease. Nature 481: 278-286, 2012.

35. Sturrock A, Cahill B, Norman K, Huecksteadt TP, Hill K, Sanders K, Karwande SV, Stringham JC, Bull DA, Gleich M, Kennedy TP, and Hoidal JR. Transforming growth factor-betal induces Nox4 NAD(P)H oxidase and reactive oxygen species-dependent proliferation in human pulmonary artery smooth muscle cells. Am J Physiol Lung Cell Mol Physiol 290: L661-L673, 2006.

36. Szabo G, and Csak T. Inflammasomes in liver diseases. $J$ Hepatol 57: 642-654, 2012.

37. Terui Y, Saito T, Watanabe H, Togashi H, Kawata S, Kamada Y, and Sakuta S. Effect of angiotensin receptor antagonist on liver fibrosis in early stages of chronic hepatitis C. Hepatology 36: 1022, 2002.

38. Thum T, Gross C, Fiedler J, Fischer T, Kissler S, Bussen M, Galuppo P, Just S, Rottbauer W, Frantz S, Castoldi M, Soutschek J, Koteliansky V, Rosenwald A, Basson MA, Licht JD, Pena JT, Rouhanifard SH, Muckenthaler MU, Tuschl T, Martin GR, Bauersachs J, and Engelhardt S. MicroRNA-21 contributes to myocardial disease by stimulating MAP kinase signalling in fibroblasts. Nature 456: 980-984, 2008.

39. Watanabe A, Sohail MA, Gomes DA, Hashmi A, Nagata J, Sutterwala FS, Mahmood S, Jhandier MN, Shi Y, Flavell RA, and Mehal WZ. Inflammasome-mediated regulation of hepatic stellate cells. Am J Physiol Gastrointest Liver Physiol 296: G1248-G1257, 2009.

40. Wenzel P, Knorr M, Kossmann S, Stratmann J, Hausding M, Schuhmacher S, Karbach SH, Schwenk M, Yogev N, Schulz E, Oelze M, Grabbe S, Jonuleit H, Becker C, Daiber A, Waisman A, and Münzel T. Lysozyme M-positive monocytes mediate angiotensin II-induced arterial hypertension and vascular dysfunction. Circulation 124: 13701381, 2011.

41. Wree A, Eguchi A, McGeough MD, Pena CA, Johnson CD, Canbay A, Hoffman HM, and Feldstein AE. NLRP3 inflammasome activation results in hepatocyte pyroptosis, liver inflammation, and fibrosis in mice. Hepatology 59: 898-910, 2014.

42. Xia Y, Jin X, Yan J, Entman ML, and Wang Y. CXCR6 plays a critical role in angiotensin II-induced renal injury and fibrosis. Arterioscler Thromb Vasc Biol 34: 14221428, 2014.

43. Yang J, Tan Y, Zhao F, Ma Z, Wang Y, Zheng S, Epstein $\mathrm{PN}, \mathrm{Yu}$ J, Yin X, Zheng Y, Li X, Miao L, and Cai L. Angiotensin II plays a critical role in diabetic pulmonary fibrosis most likely via activation of NADPH oxidasemediated nitrosative damage. Am J Physiol Endocrinol Metab 301: E132-E144, 2011.

44. Yoshiji H, Noguchi R, and Fukui H. Combined effect of an ACE inhibitor, perindopril, and interferon on liver fibrosis markers in patients with chronic hepatitis C. J Gastroenterol 40: 215-216, 2005.

45. Yoshiji H, Noguchi R, Kojima H, Ikenaka Y, Kitade M, Kaji K, Uemura M, Yamao J, Fujimoto M, Yamazaki M, Toyohara M, Mitoro A, and Fukui H. Interferon augments the anti-fibrotic activity of an angiotensinconverting enzyme inhibitor in patients with refractory chronic hepatitis C. World J Gastroenterol 12: 6786-6791, 2006.

46. Zarjou A, Yang S, Abraham E, Agarwal A, and Liu G. Identification of a microRNA signature in renal fibrosis: 
role of miR-21. Am J Physiol Renal Physiol 301: F793F801, 2011.

47. Zhang $\mathrm{X}, \mathrm{Ng}$ WL, Wang $\mathrm{P}$, Tian L, Werner E, Wang $\mathrm{H}$, Doetsch P, and Wang Y. MicroRNA-21 modulates the levels of reactive oxygen species by targeting SOD3 and TNFalpha. Cancer Res 72: 4707-4713, 2012.

48. Zhang Z, Zha Y, Hu W, Huang Z, Gao Z, Zang Y, Chen J, Dong L, and Zhang J. The autoregulatory feedback loop of microRNA-21/programmed cell death protein 4/activation protein-1 (MiR-21/PDCD4/AP-1) as a driving force for hepatic fibrosis development. J Biol Chem 288: 3708237093, 2013.

49. Zhao J, Tang N, Wu K, Dai W, Ye C, Shi J, Zhang J, Ning $\mathrm{B}$, Zeng $\mathrm{X}$, and Lin Y. MiR-21 simultaneously regulates ERK1 signaling in HSC activation and hepatocyte EMT in hepatic fibrosis. PLoS One 9: e108005, 2014.

50. Zhou R, Yazdi AS, Menu P, and Tschopp J. A role for mitochondria in NLRP3 inflammasome activation. Nature 469: 221-225, 2011.

Address correspondence to:

Prof. $\mathrm{Xu} \mathrm{Li}$

Guangdong Provincial Key Laboratory of Gastroenterology

Department of Gastroenterology Nanfang Hospital

Southern Medical University Guangzhou 510515

China

E-mail: mylx99@163.com
Date of first submission to ARS Central, February 5, 2016; date of final revised submission, July 16, 2016; date of acceptance, July 22, 2016.

$\begin{aligned} & \text { Abbreviations Used } \\ & \alpha-\mathrm{SMA}=\alpha \text {-smooth actin } \\ & \text { Ang II }=\text { angiotensin II } \\ & \text { Ang- }(1-7)=\text { angiotensin- }(1-7) \\ & \text { ASC }=\text { apoptosis-associated speck-like protein } \\ & \text { containing a caspase-recruitment domain } \\ & \text { BDL }=\text { bile duct ligation } \\ & \text { COL1A }=\alpha \text {-collagen I } \\ & \text { DAMPs }=\text { damage associated molecular patterns } \\ & \text { HSCs }=\text { hepatic stellate cells } \\ & \text { IHC }=\text { immunohistochemical } \\ & \text { ISH }=\text { in situ hybridization } \\ & \text { LPS }=\text { lipopolysaccharide } \\ & \text { mito-ROS }=\text { mitochondrial reactive oxygen species } \\ & \text { NLRP }=\text { NOD-like receptor family }, \text { pyrin domain } \\ & \quad \text { containing } 3 \\ & \text { NOX }=\text { NADPH oxidase } \\ & \text { PEG-SOD }=\text { polyethylene glycol-adsorbed-superoxide } \\ & \quad \text { dismutase } \\ & \text { q-RT-PCR }=\text { quantitative reverse-transcription } \\ & \text { polymerase chain reaction } \\ & \text { RAS }=\text { renin-angiotensin system } \\ & \text { ROS }=\text { reactive oxygen species } \\ & \text { TLR }=\text { toll-like receptor } 4 \\ & \text { VWF }=\text { von Willebrand factor }\end{aligned}$

